\title{
BEST NONPARAMETRIC BOUNDS ON DEMAND RESPONSES
}

\author{
BY RICHARD BLUNDELL, MARTIN BROWNING, AND IAN CRAWFORD ${ }^{1}$
}

This paper uses revealed preference inequalities to provide the tightest possible (best) nonparametric bounds on predicted consumer responses to price changes using consumer-level data over a finite set of relative price changes. These responses are allowed to vary nonparametrically across the income distribution. This is achieved by combining the theory of revealed preference with the semiparametric estimation of consumer expansion paths (Engel curves). We label these expansion path based bounds on demand responses as E-bounds. Deviations from revealed preference restrictions are measured by preference perturbations which are shown to usefully characterize taste change and to provide a stochastic environment within which violations of revealed preference inequalities can be assessed.

KEYWORDS: Demand responses, bounds, revealed preference, partial identification, set identification, inequality restrictions, relative prices, semiparametric regression, changing tastes.

\section{INTRODUCTION}

THIS PAPER DEVELOPS A NEW APPROACH to measuring demand responses in the study of consumer behavior. It concerns the commonly occurring empirical setting in which there is only a relatively small number of market prices but a large number of consumers within each of those markets. This research builds on the earlier results in Blundell, Browning, and Crawford (2003), where a powerful method for detecting revealed preference violations was advanced and used to provide tight nonparametric bounds on welfare costs. The contribution here is to use rich within-market consumer-level data together with the minimum of restrictions from revealed preference theory to provide the best bounds on consumer demand responses to new relative prices. These E-bounds are shown to be much tighter than those derived from standard revealed preference analysis.

A common situation in applied economics is that we have a set of observations on agents in a fixed environment with particular realized economic variables and we wish to predict their behavior in the same environment but with

\footnotetext{
${ }^{1}$ An earlier version of this paper was given as the Walras-Bowley Lecture by the first author to the North American Summer Meetings of the Econometric Society at UCLA. We are grateful to participants at that meeting, the editor, and three anonymous referees, to Adam Rosen, Rosa Matzkin, Charles Manski, and to seminar participants at Berkeley, Chicago, LSE, MIT, Northwestern, Stanford, and UCL/IFS for helpful comments. The research is part of the program of research of the ESRC Centre for the Microeconomic Analysis of Public Policy at IFS. Funding from the ESRC, Grant number R000239865 and from the Danish National Research Foundation through its Grant to CAM is gratefully acknowledged. Material from the FES made available by the ONS through the ESRC Data Archive has been used by permission of the controller of HMSO. Neither the ONS nor the ESRC Data Archive bear responsibility for the analysis or the interpretation of the data reported here. The usual disclaimer applies.
} 
new values for the economic variables. For example, we observe demands at particular sets of prices and total expenditures and we wish to predict demands at a new set of prices and total expenditure. With no other structure, the observed behavior is totally uninformative about the new situation and literally anything that is logically possible is an admissible prediction. One way around this is to use a parametric statistical model and interpolate (or extrapolate). An alternative is to adopt a theoretical position on what generates the observed behavior and to use the theory and the previous observations to make predictions. Usually this leads to bounds on predicted behavior rather than point predictions. Predicted demand responses are set-identified in the sense of Manski (2007). The relevant question then becomes "How plausible is the theory and how tight are the bounds?"

In this paper we derive bounds on predicted demand behavior from observations on expansions paths (Engel curves) for a finite set of prices and the imposition of the basic (Slutsky or revealed preference) integrability conditions from economic theory. The plausibility of the latter derives from them being, effectively, the observable restrictions from assuming transitivity, which is the bedrock of consumer theory in economics. Moreover, the theory implies testable restrictions so it is potentially rejectable. We give the tightest possible bounds on demands given observed expansion paths and the basic (nonparametric) theory, if the latter is not rejected by the former. We find that the data and the theory give surprisingly tight bounds if we consider new situations that are within the span of the observed data.

To introduce our methodology, imagine facing a set of individual consumers with a sequence of relative prices and asking them to choose their individual demands, given some overall budget that each can expend. If they behave according to the axioms of revealed preference, their vector of demands at each relative price will satisfy certain well known inequalities (see Afriat (1973) and Varian (1982)). If, for any individual, these inequalities are violated, then that consumer can be deemed to have failed to behave according to the optimization rules of revealed preference (RP). This is a very simple and potentially powerful experimental setting for assessing the applicability RP theory. If, as in an experiment, one can choose the budget at which individuals face each price vector, then Proposition 1 of Blundell, Browning, and Crawford (2003) shows that there is a unique sequence of such budgets, corresponding to the sequence of relative prices, which maximizes the chance of finding such a violation. This is the sequential maximum power path. If experimental data are not available, then the Blundell, Browning, and Crawford (2003) study also shows how to use expansion paths to mimic the experimental choice of this optimal sequence, thus providing a powerful method of detecting RP violations in observational as well as experimental studies. In this paper we extend the previous analysis in three ways. The first of these is the derivation of the tightest possible bounds on predicted demands for given relative prices and 
total outlay for observational data of the type collected in consumer expenditure surveys. To do this we find it convenient to use the strong axiom of revealed preference (SARP) rather than the more general generalized axiom of revealed preference (GARP) condition used in Blundell, Browning, and Crawford (2003). Second, we show exactly when having more data (more observed relative price regimes) is informative in the specific sense of tightening predicted bounds. The third innovation concerns how to deal with rejection of the RP conditions. We show that minimal local perturbations to the expansion paths can be found such that the perturbed data do satisfy the RP conditions. These perturbations may be interpreted as taste changes. We also discuss explicitly how our analysis relates to the important emerging literature on partial identification (see Manski (2003)).

To construct bounds we extend the analysis introduced in Varian (1983) by considering expansion paths for given relative prices rather than demands at some point. We label these "expansion path based bounds" as E-bounds. The advantages of the E-bounds method developed here are that it can describe the complete demand response to a relative price change for any point in the income distribution without recourse to parametric models of consumer behavior and it gives the tightest possible bounds, given the data and the theory. The measurements of such price responses are at the center of applied welfare economics, they are a vital ingredient of tax policy reform analysis, and are also key to the measurement of market power in modern empirical industrial economics. Robust measurement is therefore a prerequisite of reliable analysis in these fields of applied microeconomics.

Since the expansion paths are estimated, albeit by semiparametric techniques, they are subject to sampling variation. Consequently, violations of the revealed preference inequalities may simply reflect sampling variation rather than rejections by the individuals in the population under study. We develop a minimum distance method for imposing the revealed preference conditions and use this to construct a test statistic for the revealed preference inequalities. We contrast these results to those obtained using a parametric model in which Engel curves are assumed to be quadratic. We show that this parametric model produces similar results. However, the local nature of our analysis provides a persuasive case for using semiparametric Engel curves.

Examining our consumer expenditure data, we consider whether revealed preference inequality restricted expansion paths can be found that are not rejected by the data. We find that preferences are generally consistent with RP theory over subsequences of time periods in our data but that rejections over longer sequences do occur. Where significant rejections occur, there is a plethora of alternatives to the simple model which has stable preferences for the household (the unitary model). Some of these concern the supplementary assumptions we have to make on aggregation across households, aggregation of goods, the choice of an annual time period, and so forth. Other alternatives are more fundamental. For example, one alternative is that the household does 
have transitive preferences but these change over time. We present an explicit measure of such taste changes based on estimated perturbations to preferences. These provide a natural metric against which to measure taste change. Another alternative is that since our sample is for many-person households, the unitary assumption is incorrect and it is the individuals in the household who have stable transitive preferences. In this regard Browning and Chiappori (1998) presented evidence, based on a parametric model, that couples do reject the usual Slutsky conditions but not those for a nonunitary collective model. An important rationale for our RP approach is that we can be sure that any rejections of the RP conditions for the unitary model are not due to the choice of functional form. Where significant rejections do occur, the RP inequalities approach can be extended to allow for a collective model; see Cherchye, De Rock, and Vermeulen (2007).

In our empirical analysis, the relative price variation occurs over time and we consider consumer behavior as it is recorded in standard repeated consumer expenditure surveys such as the U.S. Consumers Expenditure Survey and the U.K. Family Expenditure Survey. The latter is the source for our empirical analysis. We observe samples of consumers, each of a particular household type, at specific points in time. Assuming consumers are price-takers, we can recover expansion paths by estimating Engel curves at each point in time. We present E-bounds for own- and cross-price demand responses using these expansion paths.

The E-bounds on demand responses we construct are found to be informative. The advantage of adding in more relative price variation is carefully explored, both theoretically and empirically. We show that it is the combination of the new prices and the quantity choice implied by the new expansion path that determines whether the new observation is informative. We discuss precisely how such information tightens the bounds. Empirically we show the value of allowing for sampling variation and of introducing perturbations. Bounds on demands are improved and we are also able to detect slow changes in tastes. These bounds on demand responses and the changes in tastes are found to differ across the income distribution.

Freeing up the variation in relative price responses across the income distribution is one of the key contributions of this research. Historically parametric specifications in the analysis of consumer behavior have been based on the Working-Leser or piglog form of preferences that underlie the popular almost ideal and translog demand models of Deaton and Muellbauer (1980) and Jorgenson, Lau, and Stoker (1982). Even though more recent empirical studies have suggested further nonlinear income terms (see, for example, Hausman, Newey, Ichimura, and Powell (1991), Lewbel (1991), Blundell, Pashardes, and Weber (1993), and Banks, Blundell, and Lewbel (1996)), responses to relative prices at different incomes for these parametric forms remain unnecessarily constrained.

The remainder of the paper is as follows: In Section 2 we examine bounds on demand responses and develop a method for generating the best bounds. We 
also consider how additional data impact the bounds and, in particular, the circumstance under which new data are informative. In Section 3 we describe how we apply our approach to the household level data in the U.K. Family Expenditure Survey. We examine the semiparametric estimation of expansion paths, and the method used to detect revealed preference violations and to impose revealed preference restrictions. In Section 4 we estimate E-bounds on crossprice and own-price responses, and show that these can be quite narrow. In Section 5 we consider imposing revealed preference restrictions and introduce the idea of preference perturbations. Although we find we can reject stability of preferences over the whole period from 1975 to 1999, we can find subperiods over which stable preferences cannot be rejected. This is found to substantially improve the bounds on demand responses. We also estimate bounds on demands at different percentiles of the income distribution and show that these can differ in important ways. Section 6 concludes. Online supplements contain data and programs (Blundell, Browning, and Crawford (2008)).

\section{EXPANSION PATH BOUNDS ON DEMANDS}

\subsection{Defining E-Bounds}

We shall be concerned with predicting demands given particular budgets. To this end, we assume that every agent responds to a given budget $(\mathbf{p}, x)$, where $\mathbf{p}$ is a $J$ vector of prices and $x$ is total expenditure, with a unique, positive demand $J$ vector:

AsSUMPTION U-Uniqueness of Demands: For each agent there exists a set of demand functions $\mathbf{q}(\mathbf{p}, x): \mathbb{R}_{++}^{J+1} \rightarrow \mathbb{R}_{++}^{J}$ which satisfy adding up: $\mathbf{p}^{\prime} \mathbf{q}(\mathbf{p}, x)=x$ for all prices $\mathbf{p}$ and total outlays $x$.

For a given price vector $\mathbf{p}_{t}$ we denote the corresponding $J$-valued function of $x$ as $\mathbf{q}_{t}(x)$ (with $q_{t}^{j}(x)$ for good $j$ ) and refer to this vector of Engel curves as an expansion path for the given prices. We shall also have need of the following assumption:

AsSUMPTION W-Weak Normality: If $x>x^{\prime}$, then $q_{t}^{j}(x) \geq q_{t}^{j}\left(x^{\prime}\right)$ for all $j$ and all $\mathbf{p}_{t}$.

This rules out inferior goods. Adding up and weak normality imply that at least one of the inequalities in this assumption is strict and that expansion paths are continuous.

The question we address is "Given a budget $\left\{\mathbf{p}_{0}, x_{0}\right\}$ and a set of observed prices and expansion paths $\left\{\mathbf{p}_{t}, \mathbf{q}_{t}(x)\right\}_{t=1, \ldots, T}$, what values of $\mathbf{q}$ such that $\mathbf{p}_{0}^{\prime} \mathbf{q}=\mathbf{x}_{0}$ are consistent with these observed demands and utility maximization?" Working with a finite set of observed prices, it is natural to characterize consistency 
with utility maximization in terms of revealed preference axioms. Since we are requiring that demands be single valued (and not correspondences), we work with the strong axiom of revealed preference (SARP) rather than the more usual GARP. ${ }^{2}$

Definition $1-\mathbf{q}_{t} R^{0} \mathbf{q}_{s}$ : If at prices $\mathbf{p}_{t}$ the agent chooses $\mathbf{q}_{t}$ and we have $\mathbf{p}_{t}^{\prime} \mathbf{q}_{t} \geq \mathbf{p}_{t}^{\prime} \mathbf{q}_{s}$, then we say that $\mathbf{q}_{t}$ is directly revealed weakly preferred to $\mathbf{q}_{s}$ : $\mathbf{q}_{t} R^{0} \mathbf{q}_{s}$.

Definition 2- $\mathbf{q}_{t} R \mathbf{q}_{s}$ : If we have a chain

$$
\mathbf{q}_{t} R^{0} \mathbf{q}_{u}, \mathbf{q}_{u} R^{0} \mathbf{q}_{v}, \ldots, \mathbf{q}_{w} R^{0} \mathbf{q}_{s}
$$

then we say that $\mathbf{q}_{t}$ is revealed weakly preferred to $\mathbf{q}_{s}: \mathbf{q}_{t} R \mathbf{q}_{s}$.

Given this, we have the following definition:

DEFINITION 3-SARP: $\mathbf{q}_{t} R \mathbf{q}_{s}$ and $\mathbf{q}_{t} \neq \mathbf{q}_{s}$ implies not $\mathbf{q}_{s} R^{0} \mathbf{q}_{t}$ for all $s, t$.

The definition of SARP does not rule out that we might have the same demand for two different price vectors.

The basic idea behind our analysis is shown in Figure 1 for a two-good, two expansion path example. In this example, the two expansion paths are shown as $\mathbf{q}_{1}(x)$ and $\mathbf{q}_{2}(x)$. These intersect the new budget line $\left\{\mathbf{p}_{0}, x_{0}\right\}$ at $\mathbf{q}_{1}\left(\tilde{x}_{1}\right)$ and $\mathbf{q}_{2}\left(\tilde{x}_{2}\right)$, respectively, so that

$$
\mathbf{p}_{0}^{\prime} \mathbf{q}_{1}\left(\tilde{x}_{1}\right)=\mathbf{p}_{0}^{\prime} \mathbf{q}_{2}\left(\tilde{x}_{2}\right)=x_{0} .
$$

DEFINITION 4-Intersection Demands: $\mathbf{q}_{t}\left(\tilde{x}_{t}\right)$ satisfy $\mathbf{p}_{0}^{\prime} \mathbf{q}_{t}\left(\tilde{x}_{t}\right)=\mathbf{x}_{0}$.

The two assumptions on demand above ensure that a unique intersection demand exists for any $\left\{\mathbf{p}_{0}, x_{0}\right\}$ and $\mathbf{q}_{t}(x)$. We also show the two budget lines at the intersection demands, labelled $\left\{\mathbf{p}_{1}, \widetilde{x}_{1}\right\}$ and $\left\{\mathbf{p}_{2}, \widetilde{x}_{2}\right\}$, respectively. As drawn, the two intersection demands satisfy SARP since neither is revealed weakly preferred to the other. The final step is to display the set of points on the new budget line $\left\{\mathbf{p}_{0}, x_{0}\right\}$ that are consistent with these intersection points and with SARP. This is shown as the interval labelled $S\left(\mathbf{p}_{0}, x_{0}\right)$; this set includes the intersection demands and, for two goods, it is closed. We term this set the support set for $\left\{\mathbf{p}_{0}, x_{0}\right\}$. Any point on the new budget that is in the support set $S\left(\mathbf{p}_{0}, x_{0}\right)$ satisfies SARP for the intersection demands and any point outside fails. For example, a point $\mathbf{q}_{0}$ within the interior of the support set is weakly revealed

\footnotetext{
${ }^{2}$ Varian (1982) provided a discussion of the relationship between SARP and GARP. In brief, SARP requires single-valued demand curves, whilst GARP allows for set-valued demand correspondences (so that SARP implies GARP).
} 


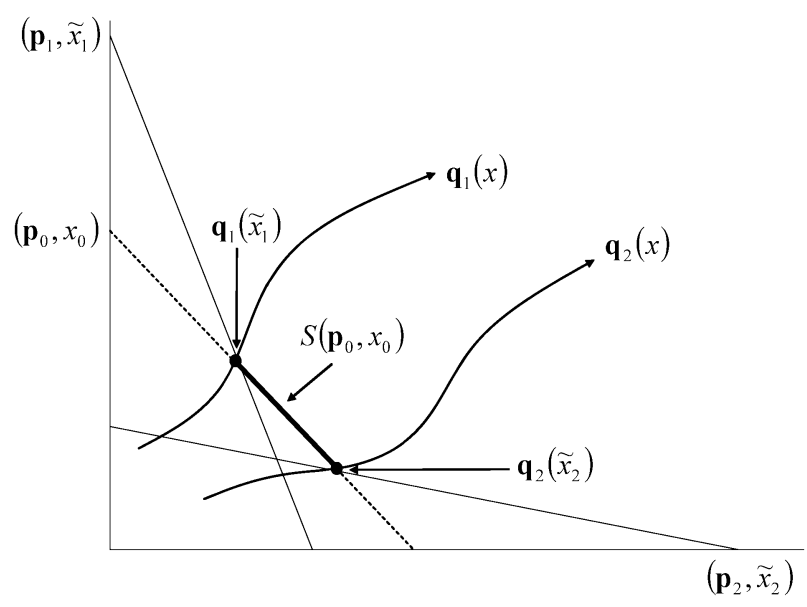

FIGURE 1.-The support set.

preferred to the intersection demands (since $\mathbf{p}_{0}^{\prime} \mathbf{q}_{0}=x_{0} \geq \mathbf{p}_{0}^{\prime} \mathbf{q}_{t}\left(\tilde{x}_{t}\right)$ for $t=1,2$ ), it is distinct from them but the intersection demands are not directly weakly preferred to $\mathbf{q}_{0}$. Conversely, consider a point $\mathbf{q}_{0}$ that is not in $S\left(\mathbf{p}_{0}, x_{0}\right)$. In this case SARP fails immediately since $\mathbf{q}_{1}\left(\tilde{x}_{1}\right) R^{0} \mathbf{q}_{0}$ (which implies $\mathbf{q}_{1}\left(\tilde{x}_{1}\right) R \mathbf{q}_{0}$ ), $\mathbf{q}_{1}\left(\tilde{x}_{1}\right) \neq \mathbf{q}_{0}$, and $\mathbf{q}_{0} R^{0} \mathbf{q}_{1}\left(\tilde{x}_{1}\right)$. Finally, the intersection points satisfy SARP and hence are in the support set.

Given Figure 1 and the definition of intersection demands, it is straightforward to define the support set algebraically. ${ }^{3}$ Given a budget $\left\{\mathbf{p}_{0}, x_{0}\right\}$, the set of points that are consistent with observed expansion paths $\left\{\mathbf{p}_{t} ; \mathbf{q}_{t}\left(\tilde{x}_{t}\right)\right\}_{t=1, \ldots, T}$ and utility maximization is given by the support set

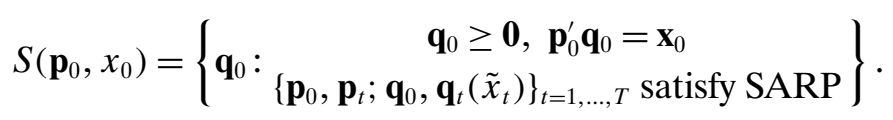

This differs from the support set definition given in Varian (1982) in two major respects. The Varian definition was based on $T$ observed demand bundles, whereas the present definition makes use of $T$ expansion paths. Furthermore, this support set is defined using expansion paths evaluated at specific budget levels: the intersection demands. We refer to the intervals defined by expansion paths in this way as E-bounds-expansion curve based demand bounds. These bounds on demands for the new budget are best in the sense that tighter bounds cannot be found without either observing more expansion paths, imposing some additional theoretical structure over and above utility maximization (such as quasi-homotheticity or separability), or assuming a functional

\footnotetext{
${ }^{3}$ In all that follows we assume that the observed prices $\left\{\mathbf{p}_{1}, \ldots, \mathbf{p}_{T}\right\}$ are relatively distinct in the sense that $\mathbf{p}_{t} \neq \lambda \mathbf{p}_{s}$ for all $s, t$ and any $\lambda>0$.
} 
form for preferences. To show this we define an alternative support set that uses points on the expansion paths that are not necessarily intersection points:

$$
S^{\prime}\left(\mathbf{p}_{0}, x_{0}\right)=\left\{\mathbf{q}_{0}: \begin{array}{c}
\mathbf{q}_{0} \geq \mathbf{0}, \mathbf{p}_{0}^{\prime} \mathbf{q}_{0}=\mathbf{x}_{0} \\
\left\{\mathbf{p}_{0}, \mathbf{p}_{t} ; \mathbf{q}_{0}, \mathbf{q}_{t}\left(x_{t}\right)\right\}_{t=1, \ldots, T} \text { satisfy SARP }
\end{array}\right\} .
$$

The next proposition states that this set is always at least as large as the support set (the proof is given in Appendix A1):

Proposition 1: If demands are weakly normal, then $S^{\prime}\left(\mathbf{p}_{0}, x_{0}\right) \supseteq S\left(\mathbf{p}_{0}, x_{0}\right)$.

Thus there do not exist alternative bounds (derived from the same data) which are tighter than the E-bounds. The E-bounds therefore make maximal use of the data and the basic nonparametric theory in predicting in a new situation. The properties of the support set are given in the following proposition:

Proposition 2: (i) $S\left(\mathbf{p}_{0}, x_{0}\right)$ is nonempty if and only if the data set $\left\{\mathbf{p}_{t}\right.$, $\left.\mathbf{q}_{t}\left(\tilde{x}_{t}\right)\right\}_{t=1, \ldots, T}$ satisfies SARP. (ii) If the data set $\left\{\mathbf{p}_{t}, \mathbf{q}_{t}\left(\tilde{x}_{t}\right)\right\}_{t=1, \ldots, T}$ satisfies $S A R P$ and $\mathbf{p}_{0}=\mathbf{p}_{t}$ for some t, then $S\left(\mathbf{p}_{0}, x_{0}\right)$ is the singleton $\left\{\mathbf{q}_{t}\left(\tilde{x}_{t}\right)\right\}$. (iii) $S\left(\mathbf{p}_{0}, x_{0}\right)$ is convex.

The first statement establishes that there are some predicted demands for $\left\{\mathbf{p}_{0}, x_{0}\right\}$ if and only if the intersection demands satisfy SARP. The second statement shows that the support set is a single point if the new price vector is one that has been observed. Our decision to consider SARP rather than GARP is largely to give this property; for GARP, we would have an interval prediction even for a previously observed price. The convexity is useful when it comes to solving numerically for E-bounds. Note that, contrary to what Figure 1 suggests, with more than two goods the support set is not necessarily closed.

The empirical analysis below requires that we compute E-bounds for given data, but the definition of $S\left(\mathbf{p}_{0}, x_{0}\right)$ is not particularly suited to empirical implementation as it stands. The second set we define gives a set of conditions that allow us to do this in a simple way using linear programming (LP) techniques. If $\left\{\mathbf{p}_{t}, \mathbf{q}_{t}\left(\tilde{x}_{t}\right)\right\}_{t=1, \ldots, T}$ satisfies SARP, we define

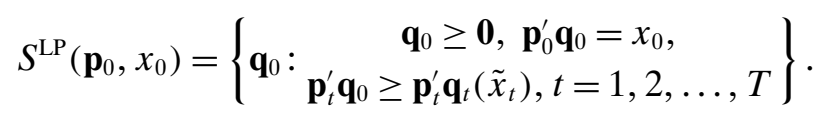

The set $S^{\mathrm{LP}}$ is closed and convex. We now show that this set is the same as the support set, except (perhaps) on the boundary of the latter. ${ }^{4}$ If we denote the closure of $S$ by $\operatorname{cl}(S)$ then we have the following proposition.

\footnotetext{
${ }^{4}$ If we had considered GARP rather than SARP, then we would have $S=S^{\mathrm{LP}}$.
} 
Proposition 3: (i) $\operatorname{cl}\left(S\left(\mathbf{p}_{0}, x_{0}\right)\right)=S^{\mathrm{LP}}\left(\mathbf{p}_{0}, x_{0}\right)$. (ii) $S^{\mathrm{LP}}\left(\mathbf{p}_{0}, x_{0}\right) \backslash S\left(\mathbf{p}_{0}, x_{0}\right)=$ $\left\{\mathbf{q} \in S^{\mathrm{LP}}\left(\mathbf{p}_{0}, x_{0}\right): \mathbf{p}_{t}^{\prime} \mathbf{q}=\tilde{x}_{t}\right.$ and $\mathbf{q} \neq \mathbf{q}_{t}\left(\tilde{x}_{t}\right)$ for some $\left.t\right\}$.

As we have seen, for two goods $S\left(\mathbf{p}_{0}, x_{0}\right)$ is closed so that it coincides with $S^{\mathrm{LP}}\left(\mathbf{p}_{0}, x_{0}\right)$, but for more than two goods the set on the right hand side of the second statement is nonempty (as long as $S\left(\mathbf{p}_{0}, x_{0}\right)$ is nonempty). $S^{\mathrm{LP}}\left(\mathbf{p}_{0}, x_{0}\right)$ gives us a feasible algorithm for displaying E-bounds. We first define intersection demands and test for SARP on the intersection demands. If the intersection demands pass SARP, we can then display bounds for each good. For example, to find the supremum predicted value for good $j$, we maximize $q_{0}^{j}$ subject to the constraints in (3). This is a standard linear programming problem.

\subsection{When Is a New Observation Informative?}

We turn now to a consideration of when and how additional observations on expansion paths lead to an improvement in our bounds. We consider the situation in which we have $T$ observed prices $\left\{\mathbf{p}_{1}, \mathbf{p}_{2}, \ldots, \mathbf{p}_{T}\right\}$. Take a hypothetical budget $\left\{\mathbf{p}_{0}, x_{0}\right\}$ and suppose that the corresponding intersection demands satisfy SARP; denote the support set by $S^{T}\left(\mathbf{p}_{0}, x_{0}\right)$. Suppose now that we add one more observed price and expansion path $\left\{\mathbf{p}_{T+1}, \mathbf{q}_{T+1}(x)\right\}$, find the corresponding intersection demand $\mathbf{q}_{T+1}\left(\tilde{x}_{T+1}\right)$, and compute the new support set $S^{T+1}\left(\mathbf{p}_{0}, x_{0}\right)$.

We begin with the following observations. First, the support set cannot increase with the introduction of a new intersection demand; that is, $S^{T+1}\left(\mathbf{p}_{0}, x_{0}\right) \subseteq S^{T}\left(\mathbf{p}_{0}, x_{0}\right)$ so that additional information weakly shrinks the support set. Second, the introduction of a new budget plane and corresponding intersection demand might cause a violation of SARP. If it does, then the new support set will be empty (by Proposition 2) and therefore, trivially, we know that the support set will strictly shrink: $S^{T+1}\left(\mathbf{p}_{0}, x_{0}\right)=\varnothing \subset S^{T}\left(\mathbf{p}_{0}, x_{0}\right)$. For the rest of this section we will set this possibility aside and assume that the new observation does not cause a violation. Given this, we ask when a new observation will be informative and lead to a strict shrinkage of the support set. The first result is trivial, but is worth formally recording.

Proposition 4: If $\mathbf{p}_{T+1}=\mathbf{p}_{0} \neq \mathbf{p}_{t}$ for $t=1, \ldots, T, S^{T}\left(\mathbf{p}_{0}, x_{0}\right)$ is nonempty, and $\mathbf{q}_{t}\left(\tilde{x}_{t}\right) \neq \mathbf{q}_{s}\left(\tilde{x}_{s}\right)$ for some $t$ and $s$, then $S^{T}\left(\mathbf{p}_{0}, x_{0}\right) \supset S^{T+1}\left(\mathbf{p}_{0}, x_{0}\right)$.

This shows that if the newly observed price just happens to coincide with $\mathbf{p}_{0}$, then the new support set will be smaller. The proof of this proposition, along with Proposition 2(ii), establishes that if the intersection points are distinct (which they will almost surely be), then the set of predicted points is a singleton only if the new price $\mathbf{p}_{0}$ is equal to one of the observed prices. More interesting is the case in which $\mathbf{p}_{T} \neq \mathbf{p}_{0}$. To present the characterization for this, we need one more definition: 
DEFINITION 5: The budget plane $\left\{\mathbf{p}_{T+1}, \tilde{x}_{T+1}\right\}$ intersects with $S^{T}\left(\mathbf{p}_{0}, x_{0}\right)$ if there exists some $\mathbf{q}_{0} \in S^{T}\left(\mathbf{p}_{0}, x_{0}\right)$ such that $\mathbf{p}_{T+1}^{\prime} \mathbf{q}_{0}=\tilde{x}_{T+1}$.

We now present conditions for strict shrinkage of the support set.

Proposition 5: Given $S^{T+1}\left(\mathbf{p}_{0}, x_{0}\right) \neq \varnothing$, then $S^{T+1}\left(\mathbf{p}_{0}, x_{0}\right) \subset S^{T}\left(\mathbf{p}_{0}, x_{0}\right)$ if and only if the new budget plane $\left\{\mathbf{p}_{T+1}, \tilde{x}_{T+1}\right\}$ intersects with $S^{T}\left(\mathbf{p}_{0}, x_{0}\right)$.

This says that a new observation is only informative in the sense that it will strictly shrink the support set if the new budget plane intersects with the initial support set. It is therefore the intersection with the initial support set which is the important feature of any new information rather than the closeness of any new price observation to the $\mathbf{p}_{0}$ vector of interest. The following three-good example serves to illustrate this proposition and to emphasize the point that if the intersection condition does not hold, then a new observation will be uninformative regardless of how close the new price vector is to the hypothetical price vector. Consider the data for three goods and three periods,

$$
\begin{aligned}
& \left\{\mathbf{p}_{1}, \mathbf{p}_{2}, \mathbf{p}_{3}\right\}=\left[\begin{array}{ccc}
0.64 & 0.19 & 0.90 \\
0.26 & 0.77 & 0.89 \\
1 & 1 & 1
\end{array}\right], \\
& \left\{\mathbf{q}_{1}, \mathbf{q}_{2}, \mathbf{q}_{3}\right\}=\left[\begin{array}{lll}
1.895 & 1.768 & 0.399 \\
1.571 & 1.141 & 1.901 \\
1.267 & 1.545 & 1.850
\end{array}\right],
\end{aligned}
$$

and take the hypothetical budget given by $\left[p_{0}^{1}, p_{0}^{2}, p_{0}^{3}\right]=[0.5,0.5,1]$ and $x_{0}=$ 3. ${ }^{5}$ Suppose now that we observe a new price $\mathbf{p}_{4}$ with an intersection demand

$$
\mathbf{q}_{4}=[1,1,2]^{\prime} .
$$

We ask "What values of $\mathbf{p}_{4}$ lead to a strict contraction of the support set?" With the values given, it is easy to show that any

$$
\mathbf{p}_{4}=\mathbf{p}_{0}-[\tau, \tau, 0]^{\prime}
$$

does not give a strict contraction for any $\tau>0$. Thus we can take an new price vector that is arbitrarily close to the hypothetical prices but does not lead to an improvement in the bounds. Conversely, any price vector

$$
\mathbf{p}_{4}=\mathbf{p}_{0}+[0, \tau, 0]^{\prime}
$$

\footnotetext{
${ }^{5}$ Note that values for the quantities have been rounded and do not exactly satisfy the intersection demand condition $\mathbf{p}_{0}^{\prime} \mathbf{q}_{t}=x_{0}$.
} 
gives a strict contraction for any $\tau>0$, even if $\tau$ is large. That is, new prices that are far from the hypothetical prices may give a strict contraction of the support set.

As we have shown, adding a new data point may tighten bounds, but it may also lead to a rejection of SARP so that more information is not an unmixed blessing. In the framework presented so far violations of SARP lead to an empty support set so that we are unable to make predictions about the demand curve. In the next section we consider how econometric estimation of expansion paths might provide a stochastic structure in which we can make progress in such a situation.

\section{ESTIMATING BOUNDS ON DEMAND RESPONSES}

\subsection{Data}

In this analysis we take three broad consumption goods-food, other nondurables, and services ${ }^{6}$ and examine the E-bounds on demand responses. For this we draw on 25 years of British Family Expenditure Surveys from 1975 to 1999. In many contexts these three consumption goods represent an important grouping as the price responsiveness of food relative to services and to other nondurables is of particular interest. For example, the price responsiveness at different income levels is a key parameter in the indirect tax debate. Although food is largely free of value added tax (VAT) in the United Kingdom, the discussions over the harmonization of indirect tax rates across Europe and the implications of a flat expenditure tax raised uniformly across all consumption items requires a good understanding of food demand responses across the income distribution. It is also important in general discussions of cost of living changes across the income distribution. Relative food prices saw some abrupt rises as the tariff structure and food import quotas were changed in Europe early in the period under study. To study further disaggregations of goods with any precision, some form of separability may have to be assumed; see Blundell, Browning, and Crawford (2007).

The Family Expenditure Survey (FES) is a repeated cross-section survey consisting of around 7,000 households in each year. From these data we draw the subsample of couples with children who own a car. This gives us between 1,421 and 1,906 observations per year and 40,731 observations over the entire period. We use total spending on nondurables to define our total expenditure variable. Table A.I in Appendix A2 provides descriptive statistics for these data. Figure 2 illustrates the trends in mean budget shares over the period. As can be seen, the mean budget share for food exhibits a large fall, whereas services are rising steadily over our data period.

${ }^{6}$ See Appendix A2. 


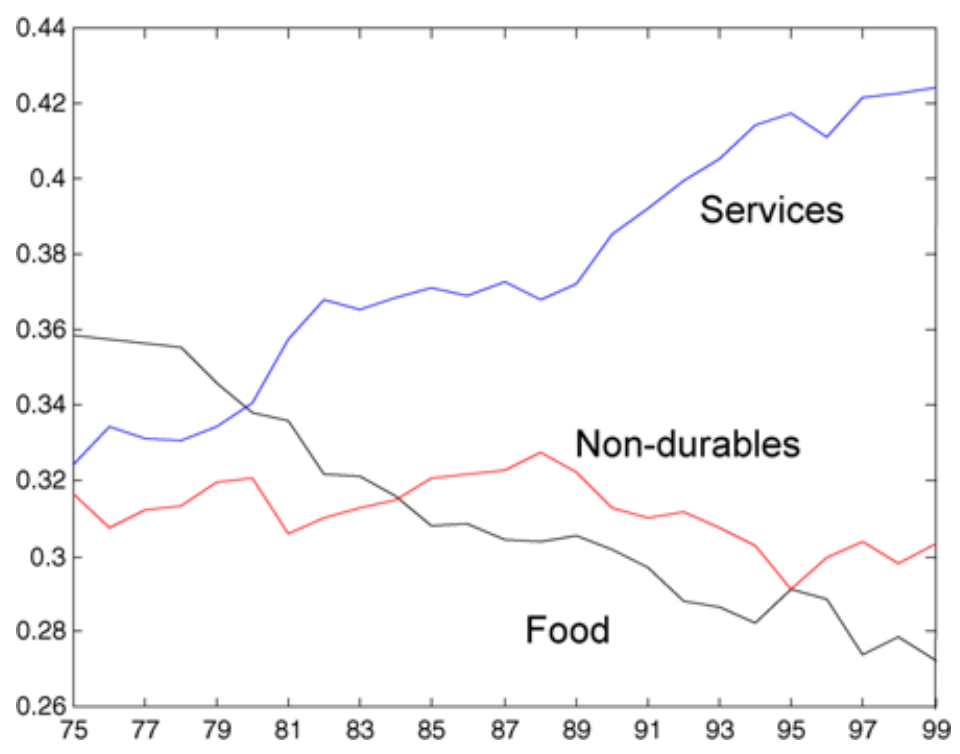

FIGURE 2.-Mean budget shares.

There was substantial relative price variation over our data period, as seen in the dated points in Figure 3. The annual price indices for these commodity groups are taken from the annual Retail Prices Index. The figure shows the scatter plot of the prices of food and services relative to nondurables. The dashed line in the figure shows the convex hull of the relative price data. The relative prices show a dramatic change in the mid to late-1970s. We see a steadily rising price for services relative to food and nondurables.

To compute the E-bounds on demand responses below we will consider variations in relative prices around a central $\mathbf{p}_{0}$ vector defined by the mean price vector. We explore a sequence of relative price changes in which the price of food is varied whilst the prices of nondurables and services are held at their mean values. The line of crosses in Figure 3 shows the particular sequence of the $\mathbf{p}_{0}$ vector we use. Note that this passes through a dense part of the relative price distribution where we might expect (subject to the discussion in Section 2) to be able to produce quite informative bounds on demand responses. The path also starts and finishes in areas of very sparse price information outside the convex hull of the prices where, without extrapolation, we would not expect to have much to say about likely demand responses. The solid lines which make up the smaller hull in Figure 3 describe a set of contiguous periods over which SARP revealed preference conditions are not rejected. We return to this case in our empirical analysis of E-bounds. We first lay out the estimation of the expansion paths and intersection demands. 


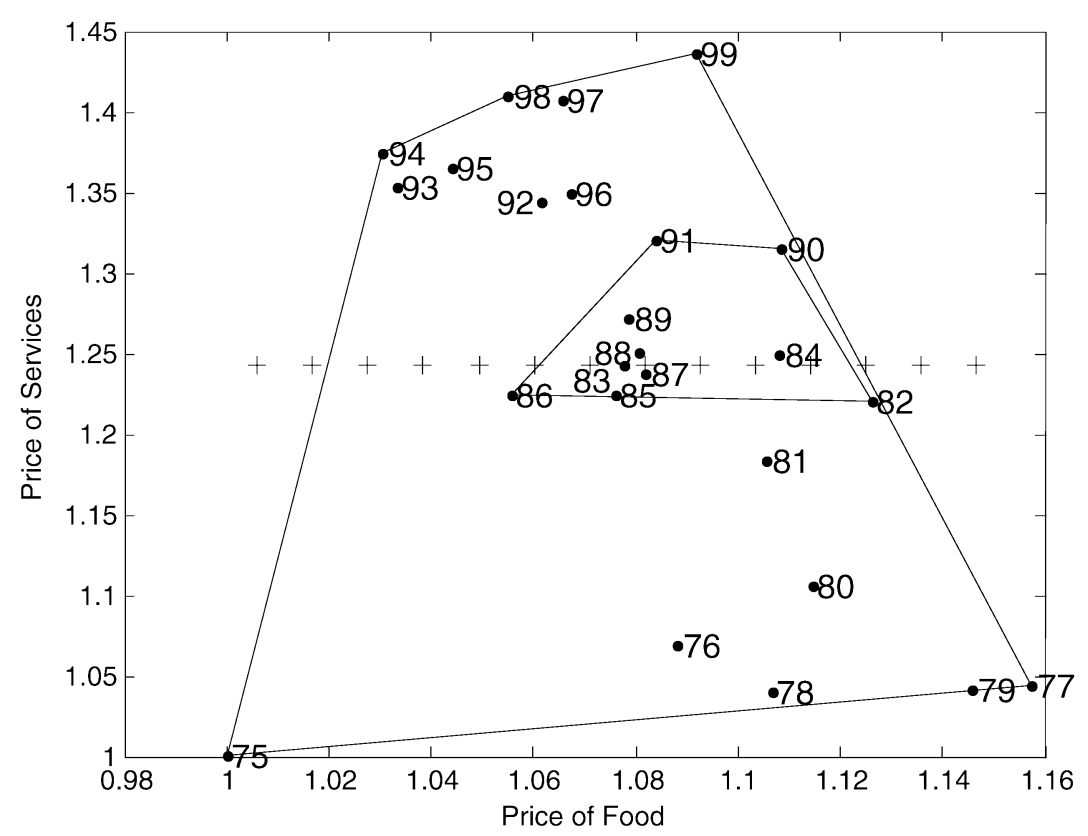

FIGURE 3.-Relative prices, 1975-1999.

\subsection{Empirical Expansion Paths}

In general, our interest is in consumer behavior described by the vector of $J-1$ demand equations

$$
q_{j}^{i}=m_{j}\left(x_{i}, \mathbf{p}, \varepsilon_{j}^{i}\right) \quad \text { for } \quad j=1, \ldots, J-1 \geq 1,
$$

where $x_{i}$ is the total outlay (or total expenditure) of household $i, \ln \mathbf{p}$ is a $J$ vector of the $\log$ of relative prices, and $\varepsilon_{j}^{i}$ is an unobservable heterogeneity term for each good $j$. We begin by assuming $\varepsilon_{j}^{i}$ to be distributed independently of $x$ and $\mathbf{p}$ but will relax the assumption on $x$.

Consumers observed in the same time period and location are assumed to face the same relative prices. Relative prices are assumed to vary exogenously. Let $\left\{\left(q_{j}^{i}, x_{i}\right)\right\}_{i=1}^{n}$ represent an independent but not identically distributed sequence of $n$ household observations on the demand $q_{j}^{i}$ of good $j$ and total expenditure $x$ for each household $i$ facing the same relative prices. Under the constant relative price assumption, Engel curves for each location and period correspond to expansion paths for each price regime. Assuming that $m_{j}$ is additively separable in $\varepsilon_{j}^{i}$ we write

$$
q_{j}^{i}=m_{j}\left(x_{i}\right)+\varepsilon_{j}^{i}
$$


for unknown continuous function $m_{j}(\cdot)$. It is these Engel curves, for each relative price regime, that we will use to estimate the intersection demands (1) in Definition 2 which are used to identify the support set (2). We will make conditional mean assumptions on the $\varepsilon_{j}^{i}$ so that the intersection demands $m_{j}\left(x_{i}\right)$ can be identified through a set of moment conditions. We will also appeal to appropriate uniform consistency and rate of convergence results from the econometric theory literature as we develop an analogous moment estimator for $m_{j}\left(x_{i}\right)$ below.

Throughout this analysis we assume the separable error form (9). As we note below, generalizations of this separable specification for unobserved heterogeneity are a key direction for future research (see Matzkin (2007) and the discussion in Section 3.2.3 below). In the estimation of these Engel curves we choose a semiparametric specification that allows for observable demographic variation in $m_{j}$ across households as well as the endogeneity of total expenditure $x$.

\subsubsection{A Semiparametric Specification for Engel Curves}

The analysis we present here is applicable to fully nonparametric specification for Engel curves. Blundell and Duncan (1998) have shown the attraction of nonparametric Engel curves when trying to capture the shape of income effects on consumer behavior across a wide range of the income distribution. However, to ensure sufficient support across the $x$ distribution, we choose the shape-invariant semiparametric specification, adopted in Blundell, Browning, and Crawford (2003), to pool across different household types.

Each household type is defined by $\mathbf{d}^{i}$, a $(D \times 1)$ vector of observable household composition variables relating to household $i=1, \ldots, n$. Our specification of (9), written in terms of budget shares $w_{j}^{i}\left(\equiv p_{j} q_{j}^{i} / x_{i}\right)$, takes the form

$$
w_{j}^{i}=g_{j}\left(\ln x_{i}-\ln \phi\left(\mathbf{d}_{i}^{\prime} \alpha\right)\right)+\mathbf{d}_{i}^{\prime} \boldsymbol{\gamma}_{j}+\xi_{j}^{i}
$$

for household $i$ and good $j$. The function $\phi\left(\mathbf{d}_{i}^{\prime} \alpha\right)$ represents a general equivalence scale and $\mathbf{d}_{i}^{\prime} \boldsymbol{\gamma}_{j}$ documents the way in which observable demographic differences $\mathbf{d}_{i}$ across households impact on each expenditure share. ${ }^{7}$ In the estimation results below we use a prior estimate of the general equivalence scale $\phi\left(\mathbf{d}_{i}^{\prime} \alpha\right)$ which we take from the Organisation for Economic Co-operation and Development (OECD) scales (Burniaux et al. (1998)). The semiparametric specification (10) turns out to be a parsimonious, yet accurate, description of behavior. ${ }^{8}$

${ }^{7}$ An expenditure share transformation is used primarily to reduce the potential for heteroscedasticity in the distribution of the $\xi_{j}$ terms. However, we only place conditional mean restrictions on these error terms and will not require homoscedasticity.

${ }^{8}$ See Blundell, Duncan, and Pendakur (1998). 
To establish the identification of the parameters of interest $g_{j}$ and $\gamma_{j}$ in this shape-invariant specification and to derive the properties of this estimator, we make the following conditional mean, continuity and bounded new assumptions:

ASSUMPTION CM: $E\left(\xi_{j}^{i} \mid \ln x_{i}, \mathbf{d}_{i}\right)=0 \forall j$.

ASSUMPTION C: $g_{j}\left(x_{i}\right)$ is continuous.

ASSUMPTION B: $w_{j}^{i}$ has bounded support.

Assumptions CM, C, and B ensure that the parameters $g_{j}$ and $\boldsymbol{\gamma}_{j}$ are identified (see Blundell, Chen, and Kristensen (2007, Theorem I)). Further, the specification chosen here, in which $\alpha$ is fixed according to an external equivalence scale, can be estimated using the partially linear regression approach in which $g_{j}$ is replaced by a Nadaraya-Watson kernel regression function (see Robinson (1988)). Results from that paper establish root- $n$ asymptotic normality and semiparametric efficiency of the parametric components $\boldsymbol{\gamma}_{j}$ and regular nonparametric convergence rates for the kernel estimator of $g_{j}$. Andrews (1995) showed uniform consistency with nonidentically distributed random variables.

\subsubsection{Endogeneity of Total Outlay $x$}

The $\ln x$ variable in (10) is a measure of log total outlay, or total expenditure, by the household on the set of goods under analysis in period $t$. This is very likely to be jointly determined with the expenditure shares. To account for the endogeneity of $\ln x$, we adopt the control function approach (see Blundell and Powell (2003) for a general discussion). We use a two-step semiparametric estimator for this model, adapting the results in Newey, Powell, and Vella (1999). The first step consists of the construction of a residual from the regression of $\ln x$ on the exogenous variables in the model and an excluded instrument. The hourly earnings of the head of household is used as the excluded instrument in the application below, where a sample of families is selected from the British FES with working age male heads of household. This control function approach is compared to the semiparametric instrumental variable estimator in Blundell, Chen, and Kristensen (2007), where it is found to account quite well for the endogeneity of total expenditure. ${ }^{9}$

The set of instrumental variables is labelled $\mathbf{z}$ and we specify the reduced form for $\ln x$ as

$$
\ln x_{i}=\mathbf{z}_{i}^{\prime} \boldsymbol{\pi}+v_{i},
$$

\footnotetext{
${ }^{9}$ Blundell, Chen, and Kristensen (2007) also considered the joint estimation of the general equivalence scale parameters $\alpha$ as well as the commodity specific parameters $\gamma$.
} 
where $\mathbf{z}$ are a set of variables which include the demographic variables $\mathbf{d}_{i}$ and the excluded instrument. ${ }^{10}$ We also make the further conditional mean assumption on $v$ which ensures the root- $n$ consistent estimation of $\pi$ from which the control function $v$ is derived:

AsSUMPTION CF1: $E\left(v_{i} \mid \mathbf{z}_{i}\right)=0$.

The control function estimator makes the following additional conditional mean assumption for the error term in (10) for each good $j$ :

ASSUMPTION CF2: $E\left(\xi_{j}^{i} \mid \ln x_{i}, \mathbf{d}_{i}, v_{i}\right)=0$.

These assumptions, together with $\mathrm{CM}, \mathrm{C}$, and $\mathrm{B}$, ensure the identification of $g_{j}$ and $\boldsymbol{\gamma}_{j}$ in (10); see Newey, Powell, and Vella (1999).

The two-step control function estimator for this model specification consists at the first step of the construction of a residual vector $\widehat{v}$ from the regression of $\ln x$ on $\mathbf{z}$. The second step is the semiparametric regression of $w_{j}$ on $g_{j}(\ln x-\ln \phi)+\mathbf{d}^{\prime} \gamma_{j}$ and $\widehat{v}$. In our estimator for (10), the additive form for this second step regression is imposed using the Robinson (1988) partially linear regression approach described above. The only further concern is the addition of the estimated term $\widehat{v}_{i}$ in each of the semiparametric regressions, based on the least squares estimate of $\pi$ in (11). Since the estimator for $\pi$ converges at a root $n$ rate, it does not effect the properties of the estimator of $g_{j}$, however, the asymptotic distribution of the estimator for $\gamma_{j}$ will depend on the distribution of $\boldsymbol{\pi}$; see Blundell and Powell (2003). Andrews (1995) showed uniform consistency and rate of convergence results for a semiparametric model of this type where the regressor variables are not observed but are based on a finite dimensional preliminary estimator as is the case here, allowing for nonidentically distributed random variables. Finally, it is worth noting that since the same variables are included on the right hand side of each of these $J-1$ Engel curves, the estimator is invariant to the equation deleted; see Blundell, Duncan, and Pendakur (1998).

\subsubsection{Nonseparable Unobserved Heterogeneity}

As we noted in the discussion of (8), our overall interest is in consumer behavior described by nonseparable heterogeneity in demands. In terms of the vector of share equations, we might express these as $\mathbf{w}=\mathbf{g}(\ln x, \ln \mathbf{p}, \mathbf{d}, \varepsilon)$, where $\varepsilon$ is a $J-1$ vector of unobservable heterogeneity. The unobserved heterogeneity enters nonseparably in the share equation. An important problem

\footnotetext{
${ }^{10}$ For the reduced form we could adopt a nonparametric specification without unduly complicating the approach used here. However, in experimentation we found it made little difference to the overall results.
} 
for future research is to estimate the distribution of demands across the heterogeneity distribution and not focus on the moments $E(w \mid \ln x, \ln \mathbf{p}, \mathbf{d})$ as we do in this paper. In the nonseparable heterogeneity case, global invertibility is required to identify the complete distribution of demands; see Brown and Matzkin (1998) and Beckert and Blundell (2005). Moreover, generalizations of quantile regression are required for estimation of the parameters of interest; see Matzkin (2007). To allow for exogeneity of $\ln x$ in such an analysis, we would need to further condition on the control variable $v$ (see Imbens and Newey (2007)).

For the more limited case of local average demands considered in this paper, there is nevertheless a general condition, due to Lewbel (2001). Under the exogeneity of $\ln x$ this condition allows interpretation to $E(w \mid \ln x, \ln \mathbf{p}, \mathbf{d})$ even in the case of nonseparable unobserved heterogeneity. If we assume $F(\varepsilon \mid \ln x, \ln \mathbf{p}, \mathbf{d})=F(\varepsilon \mid \mathbf{d})$ so that preference heterogeneity conditional on demographics is independent of prices and total outlay, then the covariance between budget shares and the responsiveness of these to changes in log total outlay, conditional on the observable determinants of demand, is defined as

$$
H(\ln x, \ln \mathbf{p}, \mathbf{d})=\operatorname{cov}\left(\frac{\partial \mathbf{g}}{\partial \ln x}, \mathbf{g}^{\prime} \mid \ln x, \ln \mathbf{p}, \mathbf{d}\right) .
$$

In this case Lewbel (2001) showed that average demands of rational consumers satisfy integrability conditions if and only if $H(\cdot)$ is symmetric and positive semidefinite. ${ }^{11}$ If $H$ is small relative to the Slutsky matrix for these average demands, then the system will be "close" to integrable. To generalize this condition to allow for the endogeneity of $\ln x$, we again need to add the control variable $v$ to write $F(\varepsilon \mid \ln x, \ln \mathbf{p}, \mathbf{d}, v)=F(\varepsilon \mid \mathbf{d}, v) .^{12}$

\section{EMPIRICAL E-BOUNDS ON DEMAND RESPONSES}

To construct E-bounds in our application to the FES data, we first estimate the three-good Engel curve system using the semiparametric control function estimator described in the previous section. Using these estimated expansion paths, we recover the estimated intersection demands $\widehat{\mathbf{q}}_{t}\left(\tilde{x}_{t}\right)$ for each $\left\{\mathbf{p}_{0}, x_{0}\right\}$ and check the revealed preference SARP conditions for $\left\{\mathbf{p}_{t}, \widehat{\mathbf{q}}_{t}\left(\tilde{x}_{t}\right)\right\}$. Perhaps unsurprisingly these unrestricted estimated intersection demands contain some violations of SARP. In the next section we develop an approach to testing and imposing the SARP conditions on the intersection demands. Before moving to that discussion, we first consider searching for contiguous periods over which we cannot reject stable preferences using the $\widehat{\mathbf{q}}_{t}\left(\tilde{x}_{t}\right)$. We find the

\footnotetext{
${ }^{11}$ For example, in the almost ideal demand system (Deaton and Muellbauer (1980)), heterogeneity in the intercept and price parameters would automatically satisfy this condition.

${ }^{12}$ We would like to thank a referee for pointing this out.
} 


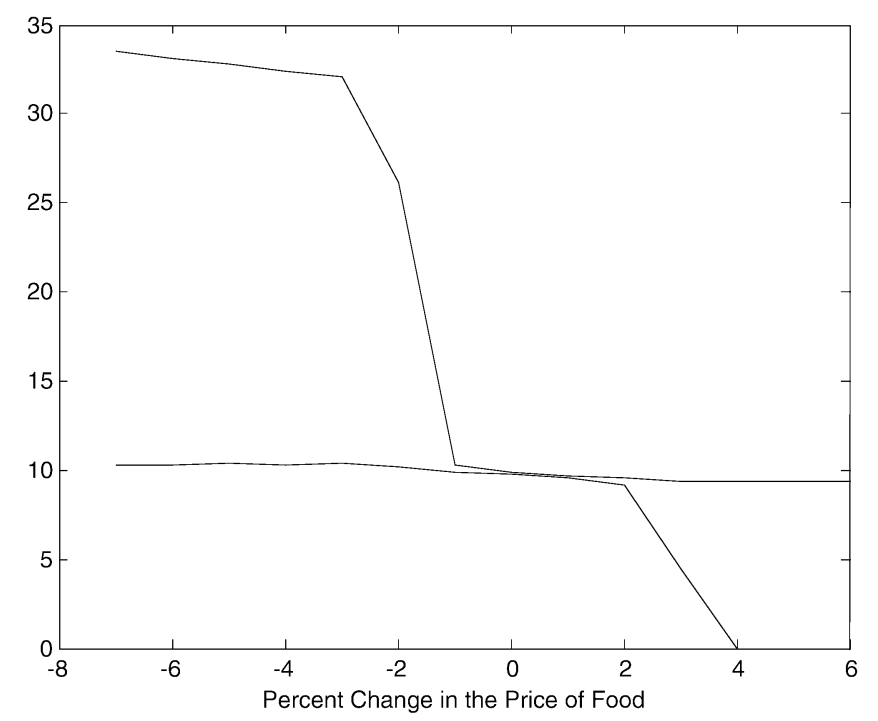

FIGURE 4.-Own-price demand bounds for food.

periods 1982 through 1991 satisfy SARP. The potential cost of discarding other periods can be seen by looking back to the smaller convex hull in Figure 3 which shows the price data corresponding to the subset of SARP-consistent intersection demands. A comparison of the two convex hulls shows the reduction in the space spanned once SARP-violating intersection demands have been dropped.

In Figure 4 we present the E-bounds on the own-price demand curve for food at the median income using the reduced set of SARP-consistent observations. As can be seen from a comparison with Figure 3, the bounds on the demand curve are particularly tight when the $\mathbf{p}_{0}$ vector is in the dense part of the observed price data. Outside the convex hull of the data the E-bounds widen and we cannot rule out extreme responses (such as households not buying food if the price rises by more than $5 \%$ ).

In Figures 5 and 6 we present the corresponding E-bounds for cross-price responses. These figures show the power of E-bounds: through the use of revealed preference inequalities and without appealing to parametric models or extrapolation we have been able to construct tight bounds on own- and crossprice responses. They also show the limitations in the sense that price experiments (by the standards typical of many policy simulation studies) can easily take on values outside the range of observed price variations and produce bounds which are necessarily very wide.

To construct E-bounds on demand curves we have exploited movements along the estimated expansion paths and it is reasonable to ask whether this involved comparisons across a wide range of incomes. In fact we find that these comparisons do not require implausibly wide variations across income levels. 


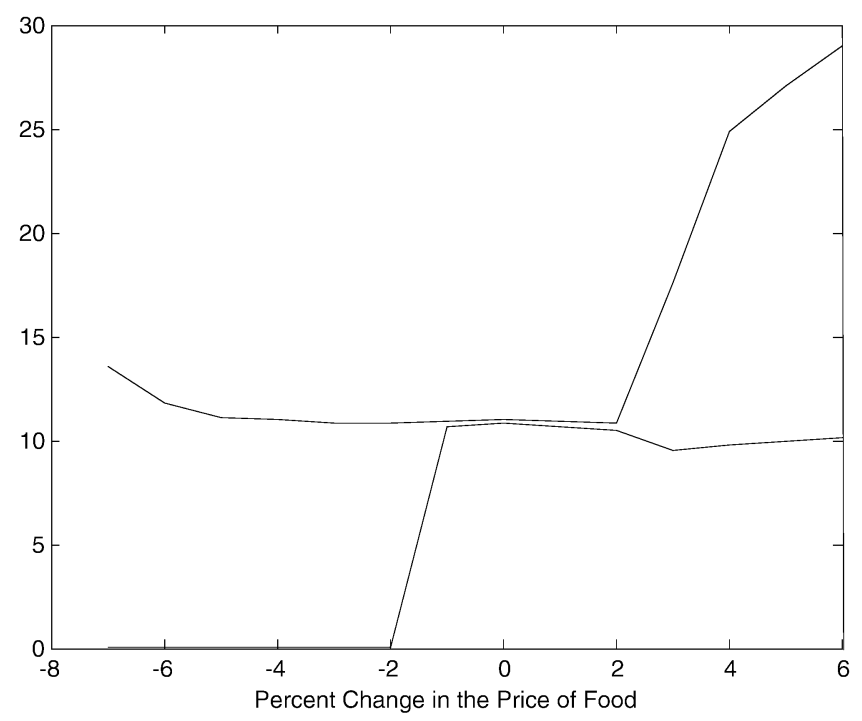

FIGURE 5.-Cross-price demand bounds for nondurables.

For example, to construct the curve in Figure 4, ten intersection demands are required. The range of income went from the 56th percentile in 1982 to the 40th percentile in 1991. This shows a further attractive feature of the local

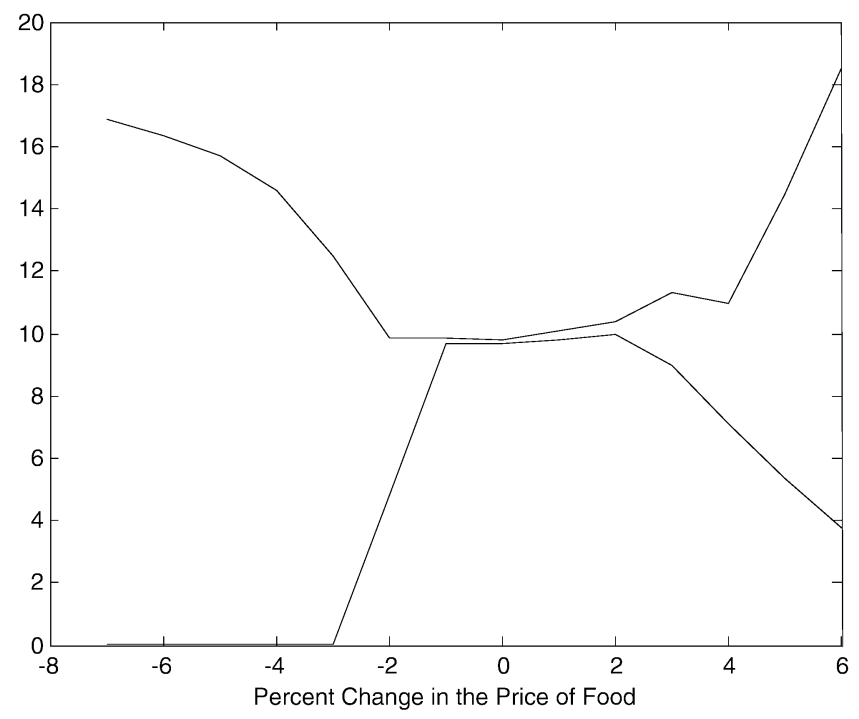

FIGURE 6.-Cross-price demand bounds for services. 
nature of this analysis: nonparametric Engel curves are only required over a limited range of the income distribution when constructing a specific demand bound at a particular income percentile.

\section{REVEALED PREFERENCE RESTRICTIONS}

\subsection{Testing and Imposing SARP}

The revealed preference restrictions, SARP in Definition 3 above, are key conditions in the identification of the E-bounds on predicted demand responses for $\left\{\mathbf{p}_{0}, x_{0}\right\}$. These bounds are defined by the support set $S\left(\mathbf{p}_{0}, x_{0}\right)$ in (2). Proposition 2 shows that this support set is non-empty (and hence identified) if and only if the data set $\left\{\mathbf{p}_{t}, \mathbf{q}_{t}\left(\tilde{x}_{t}\right)_{t=1, \ldots, T}\right\}$ satisfies SARP. Consequently, we first examine the validity of the SARP restrictions on the set of $T$ intersection demands.

Because the intersection demands are derived from the estimated expansion paths, they will be subject to sampling variation. Consequently, violations of the SARP conditions on the intersection demands may simply be due to estimation error. We use the stochastic structure of the estimated Engel curves to account for this. We derive a SARP constrained estimator for the intersection demands and a test of the SARP conditions. The starting point is the suggestion by Varian (1986) for testing optimizing behavior. We develop this idea by using the precision of the estimated expansion paths at the specific income levels that correspond to the intersection demands. We can then construct a misspecification test for violations of the revealed preference conditions.

Suppose the intersection demands, $\mathbf{q}_{t}\left(\tilde{x}_{t}\right)$, were known functions of a finite set of parameters $\boldsymbol{\theta}_{t}$ so that $\mathbf{q}_{t}\left(\tilde{\boldsymbol{x}}_{t}\right)=\mathbf{f}\left(\boldsymbol{\theta}_{t}\right)$ for known $\mathbf{f}(\cdot)$. Denote the vector of $\boldsymbol{\theta}_{t}$ 's for $t=1, \ldots, T$ by $\boldsymbol{\theta}$. In Appendix A3 we show that in the current context the SARP restrictions (see Definition 3 above) can be represented by a set of moment inequality restrictions (MIR). These place moment inequality restrictions on $\boldsymbol{\theta}$. Thus we can appeal to recent results by Manski (2003), Chernozhukov, Hong, and Tamer (2007), and Andrews and Guggenberger (2007) for moment inequality estimators of this type. There is always a value of $\boldsymbol{\theta}$ that satisfies the MIR as long as the support of the estimated $\boldsymbol{\theta}$ values allows for any positive demands that satisfy adding up..$^{13}$ Generally there will be a set of values for $\boldsymbol{\theta}$ that satisfy SARP. This set may include the intersection demands, in which case the latter satisfy SARP.

Let $\mathbb{S}$ denote the set of all intersection demands that satisfy SARP. The support set that corresponds to any set of intersection demands contained in $\mathbb{S}$ is unique and convex. If the SARP conditions fail for the unrestricted intersection demands $\widehat{\mathbf{q}}_{t}\left(\tilde{x}_{t}\right)_{t=1, \ldots, T}$, we generate a restricted estimator $\widehat{\mathbf{q}}_{t}^{\mathbb{S}}$ using the

\footnotetext{
${ }^{13}$ In that case we could take, for example, the demands implied by a Cobb-Douglas utility function with equal weights for each good. We use these demands as starting values for the minimum distance problem described below.
} 
Gaussian quasi-likelihood ratio or minimum distance criterion function

$$
\begin{aligned}
& \mathbb{Q}=\min _{\left\{\mathbf{q} t_{t=1, \ldots, T}\right.} \sum_{t=1}^{T}\left(\mathbf{q}_{t}-\widehat{\mathbf{q}}_{t}\left(\widetilde{x}_{t}\right)\right)^{\prime} \Omega_{t}^{-1}\left(\mathbf{q}_{t}-\widehat{\mathbf{q}}_{t}\left(\widetilde{x}_{t}\right)\right) \\
& \text { subject to }\left\{\mathbf{q}_{t}\right\}_{t=1, \ldots, T} \in \mathbb{S},
\end{aligned}
$$

where the weight matrix $\Omega_{t}^{-1}$ is the inverse of the covariance matrix of the estimated unrestricted intersection demands $\widehat{\mathbf{q}}_{t}\left(\widetilde{x}_{t}\right)$. The solution to (12) defines intersection demands $\widehat{\mathbf{q}}_{t}^{\mathbb{S}}$ which satisfy SARP and are unique almost everywhere.

Evaluated at the restricted intersection demands, the distance function (12) also provides a test statistic for SARP. To investigate the properties of this test, recall that the SARP restrictions can be represented as a set of moment inequality restrictions; see Appendix A3. Consequently, this test falls within the general class of misspecification tests investigated in Andrews and Guggenberger (2007, Section 7). We note however that their results are not directly applicable as they are derived for a parametric specification, as in $\mathbf{q}_{t}\left(\tilde{x}_{t}\right)=\mathbf{f}\left(\boldsymbol{\theta}_{t}\right)$ above, whereas we adopt a semiparametric specification for $\mathbf{q}_{t}\left(\tilde{x}_{t}\right)$ in our application. We conjecture that, given uniform consistency of the estimator for the intersection demands, the results will carry through to the semiparametric case, but we leave this to future research. For comparison, in Appendix A4, we present a set of estimated bounds using a fully parametric specification for the intersection demands.

To construct critical values, we let $n_{t}$ denote the sample size in period $t$ and draw samples of size $b_{n_{t}}<n_{t}$ with replacement. We assume $b_{n_{t}} \longrightarrow \infty$ and $b_{n_{t}} / n_{t} \longrightarrow 0$ as $n_{t} \longrightarrow \infty$. The subsample statistics used to construct the $1-$ $\alpha$ sample quantile are then defined exactly as in (12), but are based on the subsample of size $b_{n_{t}}$ rather than the full sample. We repeat this many times and obtain the empirical distribution of $\mathbb{Q}$. We denote the $1-\alpha$ quantile of the distribution as $\mathbb{C}_{1-\alpha}$. The nominal level $\alpha$ test rejects the SARP restrictions if and only if the statistic $\mathbb{Q}$ exceeds the subsampling critical value $\mathbb{C}_{1-\alpha}$. This test statistic can also be used to define a confidence set for the identified support set $S\left(\mathbf{p}_{0}, x_{0}\right)$ for the predicted demands $\mathbf{q}_{0}$. This draws on the recent work by Chernozhukov, Hong, and Tamer (2007) and references therein. ${ }^{14}$

An interpretation of the restricted intersection demands $\widehat{\mathbf{q}}_{t}^{\mathrm{S}}$ is as locally perturbed demands that conform to SARP. That is, they measure the minimum perturbation to tastes necessary to ensure preference stability. Consequently, the estimated "perturbations" $\widehat{\mathbf{q}}_{t}^{\Im}-\widehat{\mathbf{q}}_{t}\left(\widetilde{x}_{t}\right)$ themselves are likely to be of interest: random taste behavior would be reflected in a corresponding random

\footnotetext{
${ }^{14}$ Andrews and Guggenberger (2007) considered the subsampling confidence set for set identified parameters.
} 
pattern in perturbations; slowly changing tastes would be reflected by a systematic evolution of these perturbations. We analyze the estimated perturbations in our empirical analysis below.

\subsection{Constrained E-Bounds}

In Section 4 we searched for a contiguous period of SARP-consistent demands and simply discarded those intersection demands which caused violations. In this section we investigate the improvements in the E-bounds which can be made if we impose SARP consistency on the intersection demands across relative prices even where violations occur. We do this using the minimum distance criterion function (12). In principle this should further tighten the bounds because (i) it will expand the convex hull of the prices in use, thereby potentially increasing the range over which we can tightly bound the demand curves, and (ii) the extra information may include budget planes which intersect with the support sets which underlie Figures 4, 5, and 6. By Proposition 5 this will strictly shrink the bounds.

We begin our examination of the constrained E-bounds by imposing SARP at all the observed relative prices $t=1, \ldots, T$. To do this we use the inverse of the estimated pointwise variance-covariance matrix of the estimated expansion paths (evaluated at the intersection demands values) as the weight matrix $\Omega_{t}^{-1}$ in the minimum distance procedure (12). Tests for the restrictions are presented below. This produces a sequence of estimated restricted intersection demands $\widehat{\mathbf{q}}_{t}^{\mathrm{S}}$ and analogous perturbations $\widehat{\mathbf{q}}_{t}^{\mathrm{S}}-\widehat{\mathbf{q}}_{t}\left(\widetilde{x}_{t}\right)$.

Figure 7 presents the estimated perturbations $\widehat{\mathbf{q}}_{t}^{S}-\widehat{\mathbf{q}}_{t}\left(\widetilde{x}_{t}\right)$ by good and period at the median income. Since there are three goods and 25 intersection demands (one each of the 25 annual Engel curves), there are 75 perturbations. No structure is imposed on these perturbations other than that the restricted intersection demands are nonnegative and satisfy SARP. The figure also contains $95 \%$ pointwise confidence intervals which suggest an extended period where we may be able to find a stable representation of preferences.

If demand behavior were completely random or if it were rational but contaminated with classical measurement error, then we might expect that the perturbations would reflect this. Slowly changing tastes, on the other hand, would be reflected by a systematic evolution of these perturbations. In fact, the adjustments needed to make these data theory-consistent seem to follow a reasonably systematic pattern. The perturbation to food demand, for example, is generally increasing over time. It is negative in the early data, indicating that the earlier food demands need to be adjusted downward and the later observations need to be adjusted upward. This can be interpreted as the perturbation necessary to adjust for a slow change in preferences away from food and toward services. Comparing this to Figure 2, we can see that this adjustment would go some way to slowing the apparent decline in the food share over the period. More significantly, this analysis shows that we cannot rationalize the 


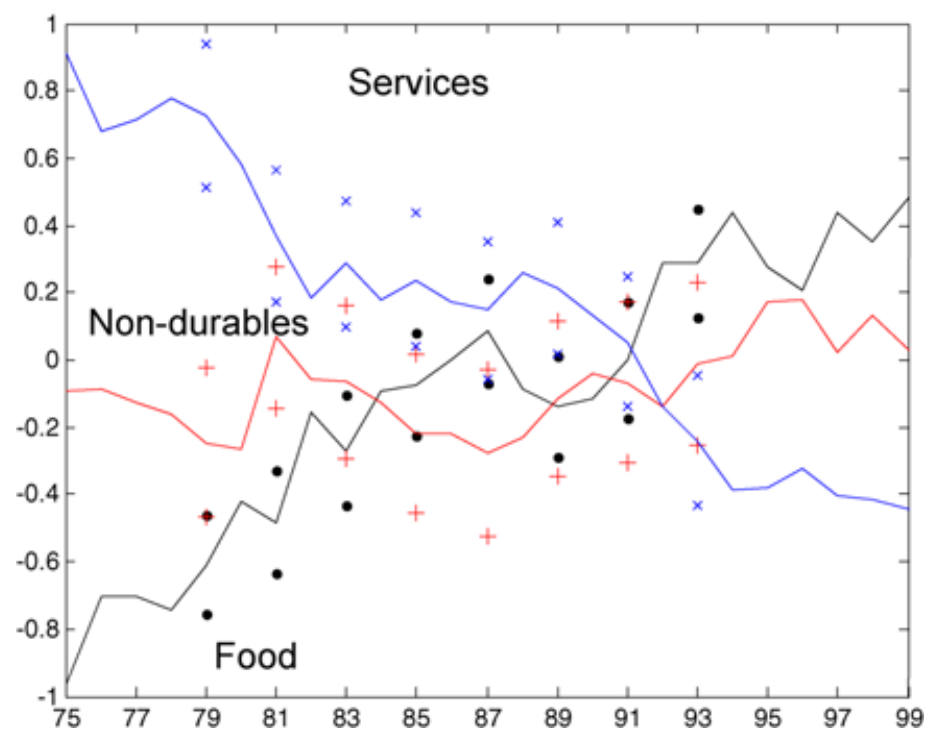

FIGURE 7.-Demand perturbations.

changes in mean budget shares seen in Figure 2 by appealing to price, income, or demographic changes.

The restricted intersection demands satisfy SARP and so we can identify the support set $S\left(\mathbf{p}_{0}, x_{0}\right)$, which deliver the E-bounds for the predicted demand responses at $\left\{\mathbf{p}_{0}, x_{0}\right\}$. The resulting estimated E-bounds on the own-price demand curve are illustrated in Figures 8 and 9 along with, for comparison, the E-bounds recovered by dropping SARP rejections (Figure 4: the solid lines). As can be seen, there is an improvement/narrowing of the bounds when all of the observations are used and constrained to be revealed preference consistent, compared to the case in which some data points are just dropped. Nevertheless, the improvement is quite small in the central part of the demand curve (see Figure 9) where the existing bounds were already fairly tight. Note also that there is no reason for the new bounds to lie everywhere inside the old bounds. Whilst the addition of theory-consistent data always weakly tightens the bounds, the data being added here contain violations and have been perturbed as a result. Consequently the restricted intersection demands can lead to the bounds widening at some relative price points. The general patterns of the bounds are similar, however, with typically wider bounds the further the new price vector is from the most dense part of the observed price distribution.

As before it is useful to examine the range of incomes (total budgets) over which comparisons have been made to construct these E-bounds for the median income consumer. Again the range is quite limited going from a maximum of the 60 percentile in the mid-1970s to the 40th percentile at the end of the 1990s. 


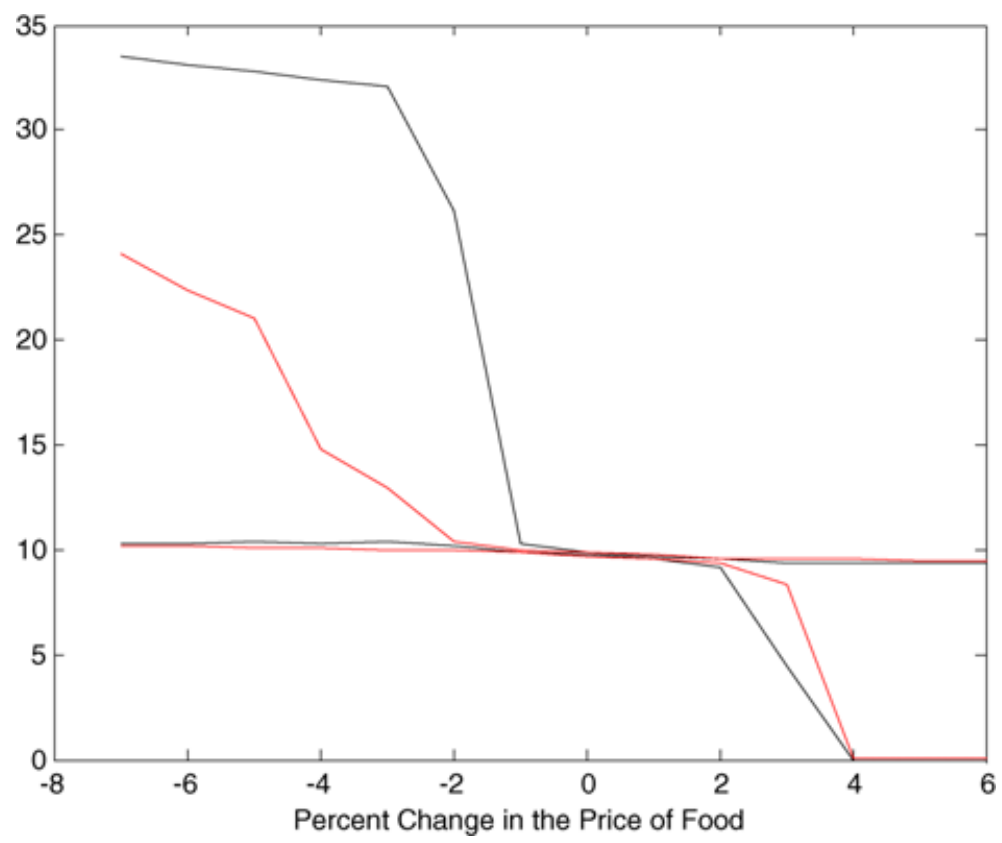

FIGURE 8.-Constrained E-bounds for food.

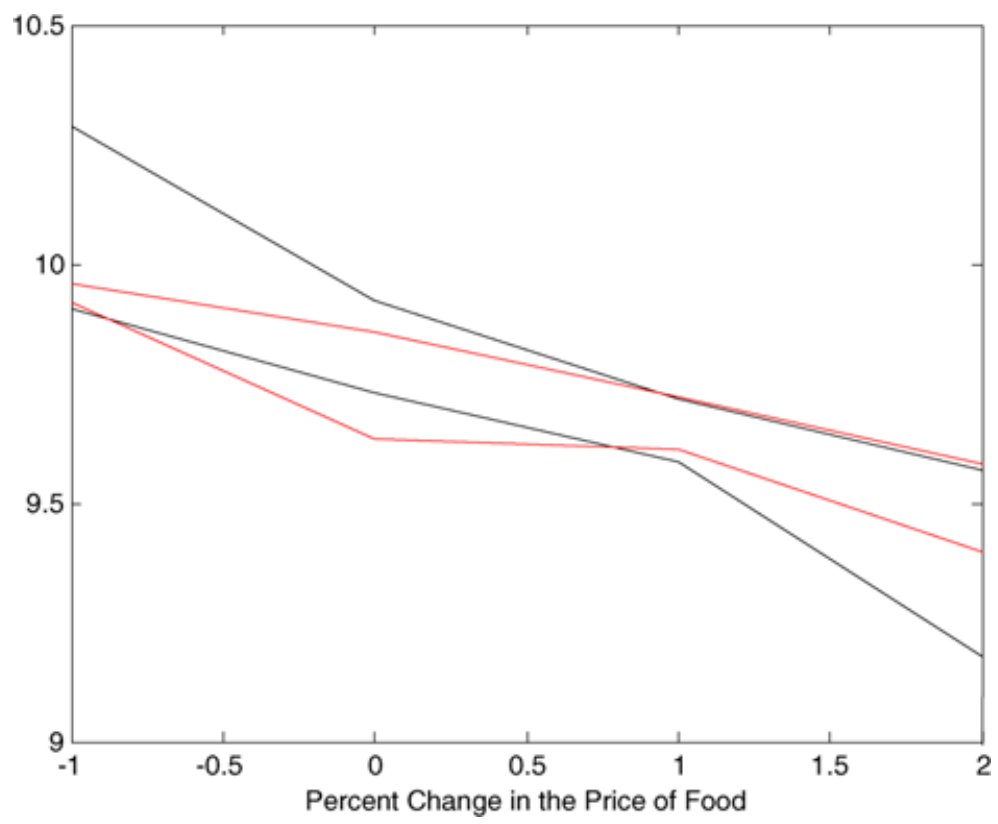

FIGURE 9.-Constrained E-bounds for food: detail. 


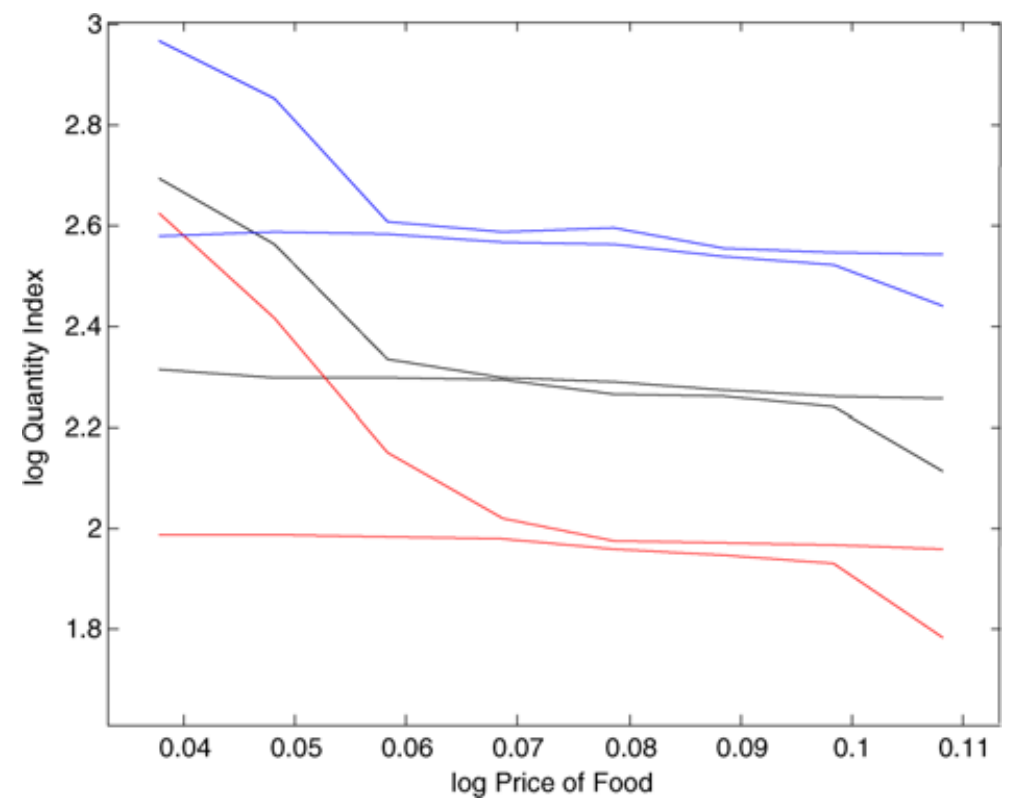

FIGURE 10.-Demand bounds for food by budget percentile (log-log).

\subsection{Demand Responses Across the Income Distribution}

The E-bounds on predicted demands presented above have been constructed at the median income (expenditure), but we might expect demand responses to vary with income levels. Figure 10 shows how the demand bounds vary according to the total budget. Three sets of bounds are calculated, corresponding to the 25th, 50th, and 75th percentiles of the $x_{0}$ distribution (the solid lines for the median are identical to the dashed lines in the preceding figure over this range). It is clear from this figure that there is not a single elasticity that summarizes price response behavior. Price responses appear to be quite variable both along each demand curve and across income levels. The range of price responsiveness highlights the local nature of our nonparametric analysis. The price responsiveness is local to both income and relative prices. Unlike in the Stone-Geary model, for example, there is no reason why price elasticities should not be increasing or decreasing with income. For some broad aggregates such as food, a price elasticity which is increasing with income would seem sensible, while for more disaggregated food items - rice and potatoes, for example-the reverse could equally well be true.

\subsection{Revealed Preference Violations and Best RP-Consistent Demands}

In the analysis so far we have investigated two approaches to dealing with violations of revealed preference on the intersection demands $q_{t}\left(\tilde{x}_{t}\right)_{t=1, \ldots, T}$ : 


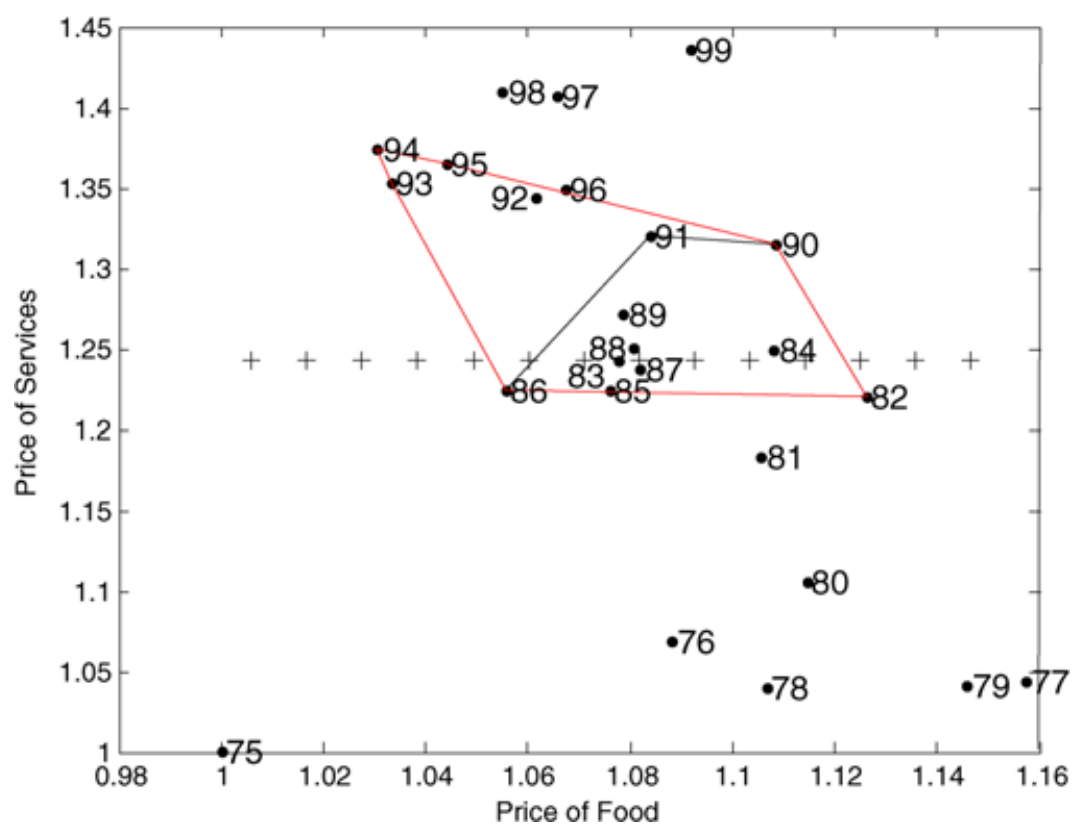

FIGURE 11.-Price scatter plot of the extended period.

dropping offending intersection demands and imposing SARP restrictions on all of the data using the minimum distance criterion (12). We have seen that the perturbations required to make the intersection demands SARP-consistent are trended and are consistent with a story of systematic taste change over the period. However, we can also use (12) to test the SARP restrictions. As described in Section 5.1, we adopt a subsampling critical value with $b_{n_{t}} / n_{t}=0.2$ which convincingly rejects SARP with a $p$-value very close to zero. ${ }^{15}$ Simply imposing SARP across the intersection demands in all the periods in the data $q_{t}\left(\tilde{x}_{t}\right)_{t=1, \ldots, T}$ is clearly invalid.

We therefore return to our SARP-consistent data set described in Figure 3. As the perturbations in Figure 7 suggest, it may be possible to add intersection demands outside this period without rejecting the SARP restrictions. Using the criteria (12), we found that expanding the set of intersection demands by adding the periods 93-95 did not reject SARP: the 20\% $\left(b_{n_{t}} / n_{t}=0.2\right)$ subsample $p$-value was 0.08 . The extended convex hull of the relative price space spanned by these periods is shown in Figure 11.

Using this extended set of SARP-consistent intersection demands results in the E-bounds on the own-price demand curve in Figures 12 and 13. Fig-

\footnotetext{
tively).

${ }^{15}$ Rejection also occurs using a $25 \%$ and a $15 \%$ subsample $\left(b_{n_{t}} / n_{t}=0.25\right.$ and 0.15 , respec-
} 


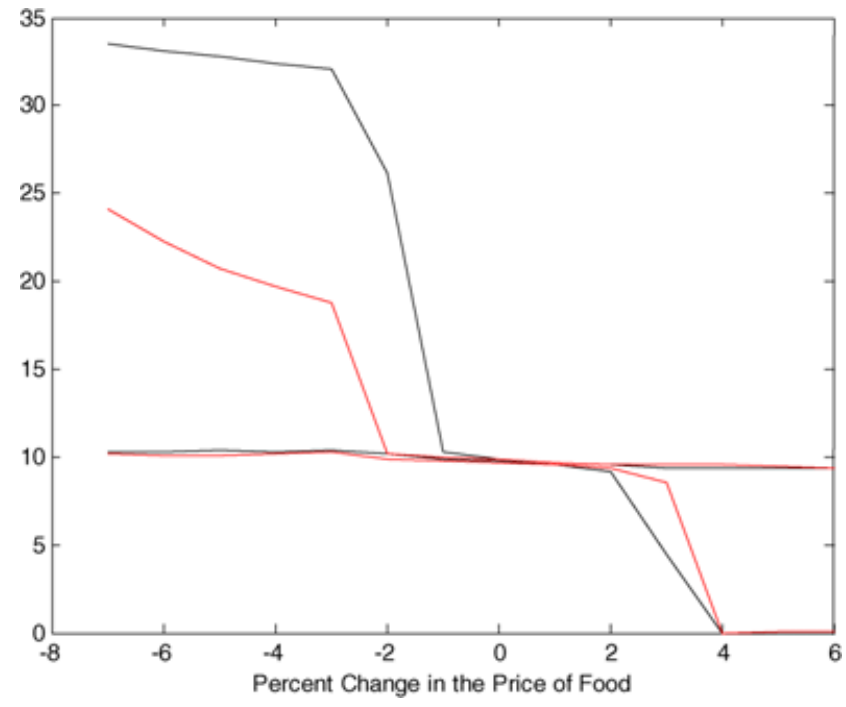

FIGURE 12.-Best RP-consistent E-bounds for food.

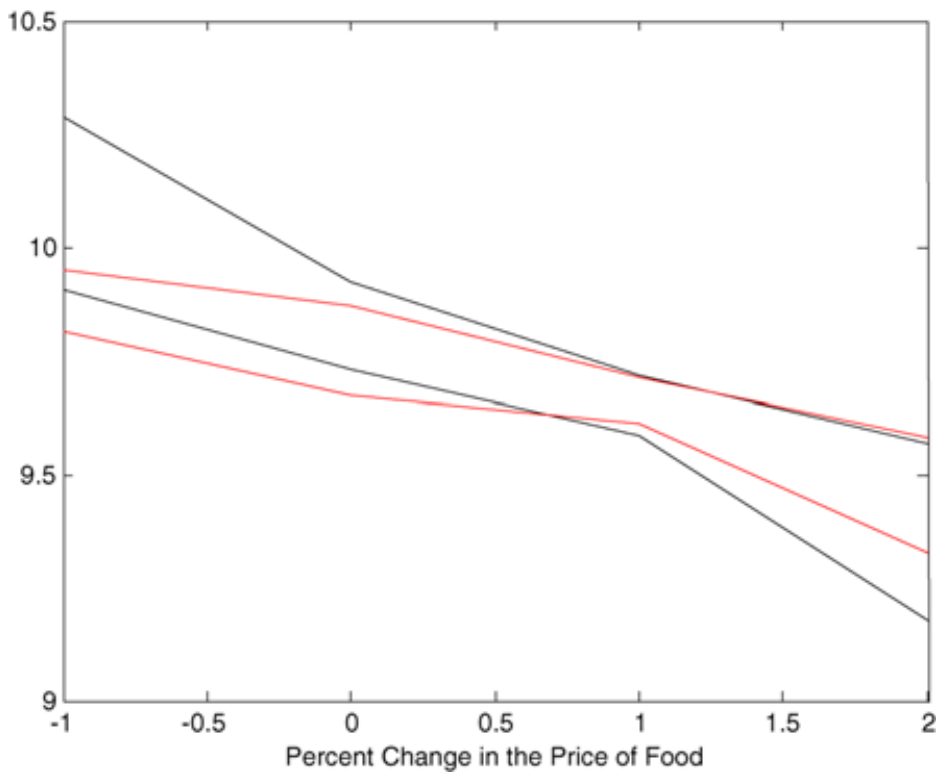

FIGURE 13.-Best RP-consistent E-bounds for food: detail. 
ure 12 shows the demand curve using the original SARP-consistent subset of the data (solid lines) and the demand curve obtained by imposing SARP on the extended demand subset of the data. For comparison, in Appendix A4 we present results that use the parametric quadratic almost ideal demand system (QUAIDS) specification for the expansion paths. These results are similar to the semiparametric case and, as expected, display even tighter bounds on demand responses. However, given the local nature of our revealed preference analysis, we choose to present the results that relate to the semiparametric Engel curve analysis in the main text of this paper, leaving the parametric analysis to the Appendix.

Figure 13 gives a detailed view of the central part of the demand curve. At "zero" the E-bounds using the extended period are [9.6742, 9.8694]. These bounds are quite precise and the $\left(b_{n} / n=0.2\right)$ subsample $95 \%$ confidence set is $[9.4987,9.9516]$. As in our discussion of Figure 8, the extended period uses restricted intersection demands and there is no requirement that the new Ebounds lie everywhere inside the bounds that simply use the 82-91 period.

\section{SUMMARY AND CONCLUSIONS}

The aim of this paper has been to bound predictions of demand responses using revealed preference inequalities. We have focussed on the situation where we can observe only a relatively small number of market prices but a large number of consumers in each of these markets. Our approach has been to make use of this rich within-market consumer-level data to estimate income expansion paths conditional on prices. We have shown how to derive best bounds on predicted demand behavior from a combination of observations on expansion paths and the imposition of the basic (Slutsky or revealed preference) integrability conditions from economic theory. We find that these E-bounds give surprisingly tight bounds, especially where we consider new situations that are within the span of the relative price data in observed markets.

The E-bounds approach to measuring consumer behavior allows price responses to vary nonparametrically across the income distribution by exploiting microdata on consumer expenditures and incomes over a finite set of discrete relative price changes. We have introduced the concept of preference perturbations, local to each income percentile, which characterize the degree of congruence with RP conditions and provide a useful metric for describing taste change.

\section{APPENDIX A1: PROOFS OF PROPOSITIONS}

Proof of Proposition 1: Let $S^{\prime}\left(\mathbf{p}_{0}, x_{0}\right)$ denote the support set

$$
S^{\prime}\left(\mathbf{p}_{0}, x_{0}\right)=\left\{\begin{array}{c}
\mathbf{p}_{0}^{\prime} \mathbf{q}_{0}=x_{0}, \mathbf{q}_{0} \geq \mathbf{0} \text { and } \\
\mathbf{q}_{0}:\left\{\mathbf{p}_{0}, \mathbf{p}_{t} ; \mathbf{q}_{0}, \mathbf{q}_{t}(x)\right\}_{t=1, \ldots, T} \text { satisfies SARP } \\
\text { and } x_{t} \neq \tilde{x}_{t} \text { for some } t
\end{array}\right\},
$$


where the $\mathbf{q}_{t}(x)$ data are demands on expansion paths at arbitrary budget levels. Suppose that there exists some demand vector $\mathbf{q}_{0} \geq \mathbf{0}$ and $\mathbf{p}_{0}^{\prime} \mathbf{q}_{0}=x_{0}$ such that $\mathbf{q}_{0} \in S\left(\mathbf{p}_{0}, x_{0}\right)$ but $\mathbf{q}_{0} \notin S^{\prime}\left(\mathbf{p}_{0}, x_{0}\right)$. Then by definition of $S^{\prime}\left(\mathbf{p}_{0}, x_{0}\right)$ it must be the case that $\left\{\mathbf{p}_{0}, \mathbf{p}_{t} ; \mathbf{q}_{0}, \mathbf{q}_{t}(x)\right\}_{t=1, \ldots, T}$ contains a violation of SARP. That is, there is some element of $\left\{\mathbf{q}_{t}(x)\right\}_{t=1, \ldots, T}$ (call it $\left.\mathbf{q}_{t}(x)\right)$ such that either $\mathbf{q}_{t}(x) R \mathbf{q}_{0}$ and $\mathbf{q}_{0} R^{0} \mathbf{q}_{t}(x)$ or $\mathbf{q}_{0} R \mathbf{q}_{t}(x)$ and $\mathbf{q}_{t}(x) R^{0} \mathbf{q}_{0}$. Consider the first case where $\mathbf{q}_{0} R^{0} \mathbf{q}_{t}(x)$. If demands are weakly normal, then the corresponding intersection demand $\mathbf{q}_{t}\left(\tilde{x}_{t}\right)$ used to define $S\left(\mathbf{p}_{0}, x_{0}\right)$ must be such that $\mathbf{q}_{t}\left(\tilde{x}_{t}\right) R^{0} \mathbf{q}_{t}(x)$. But $\mathbf{q}_{t}(x) R \mathbf{q}_{0}$ and hence $\mathbf{q}_{t}(x) R \mathbf{q}_{t}\left(\tilde{x}_{t}\right)$, and there is a contradiction of SARP. Now consider the second case where $\mathbf{q}_{t}(x) R^{0} \mathbf{q}_{0}$. Since $\mathbf{q}_{0} \in S\left(\mathbf{p}_{0}, x_{0}\right)$ we know that by definition $\mathbf{p}_{t}^{\prime} \mathbf{q}_{0} \geq \mathbf{p}_{t}^{\prime} \mathbf{q}_{t}\left(\tilde{x}_{t}\right)$ and hence $\mathbf{q}_{t}(x) R^{0} \mathbf{q}_{t}\left(\tilde{x}_{t}\right)$. Therefore, we have another contradiction of SARP. Hence $\mathbf{q}_{0} \notin S^{\prime}\left(\mathbf{p}_{0}, x_{0}\right) \Rightarrow \mathbf{q}_{0} \notin S\left(\mathbf{p}_{0}, x_{0}\right)$.

Q.E.D.

Proof of Proposition 2: (i) $S\left(\mathbf{p}_{0}, x_{0}\right)$ is nonempty if and only if the data set $\left\{\mathbf{p}_{t}, \mathbf{q}_{t}\left(\tilde{x}_{t}\right)\right\}_{t=1, \ldots, T}$ satisfies SARP. If $\left\{\mathbf{p}_{t}, \mathbf{q}_{t}\left(\tilde{x}_{t}\right)\right\}_{t=1, \ldots, T}$ fails SARP, then so does $\left\{\mathbf{p}_{0}, \mathbf{p}_{t} ; \mathbf{q}_{0}, \mathbf{q}_{t}\left(\tilde{x}_{t}\right)\right\}_{t=1, \ldots, T}$ for any $\left\{\mathbf{p}_{0} ; \mathbf{q}_{0}\right\}$ so that the support set is empty. Conversely, if $\left\{\mathbf{p}_{t}, \mathbf{q}_{t}\left(\tilde{x}_{t}\right)\right\}_{t=1, \ldots, T}$ passes SARP, then these points satisfy the conditions for inclusion in $S\left(\mathbf{p}_{0}, x_{0}\right)$ which is thus nonempty.

(ii) $S\left(\mathbf{p}_{0}, x_{0}\right)$ is the singleton $\mathbf{q}_{t}\left(\tilde{x}_{t}\right)$ if $\mathbf{p}_{0}=\mathbf{p}_{t}$ and the data set $\left\{\mathbf{p}_{t}\right.$, $\left.\mathbf{q}_{t}\left(\tilde{x}_{t}\right)\right\}_{t=1, \ldots, T}$ satisfies SARP. Let $\mathbf{p}_{0}=\mathbf{p}_{t}$ and suppose there is a $\mathbf{q}_{0} \in S\left(\mathbf{p}_{0}, x_{0}\right)$ with $\mathbf{q}_{0} \neq \mathbf{q}_{t}\left(\tilde{x}_{t}\right)$. We have $\mathbf{p}_{0}^{\prime} \mathbf{q}_{0}=x_{0}$. By construction $\mathbf{q}_{t}\left(\tilde{x}_{t}\right) R^{0} \mathbf{q}_{0}$, which implies $\mathbf{q}_{t}\left(\tilde{x}_{t}\right) R \mathbf{q}_{0}$. Since $\mathbf{q}_{0}$ satisfies SARP and $\mathbf{q}_{0} \neq \mathbf{q}_{t}\left(\tilde{x}_{t}\right)$, we have $\operatorname{not}\left(\mathbf{q}_{0} R^{0} \mathbf{q}_{t}\left(\tilde{x}_{t}\right)\right)$ which is equivalent to $\mathbf{p}_{0}^{\prime} \mathbf{q}_{0}<\mathbf{p}_{0}^{\prime} \mathbf{q}_{t}\left(\tilde{x}_{t}\right)=\mathbf{p}_{t}^{\prime} \mathbf{q}_{t}\left(\tilde{x}_{t}\right)$. Since both sides of this strict inequality are equal to $x_{0}$, this gives a contradiction.

(iii) $S\left(\mathbf{p}_{0}, x_{0}\right)$ is convex. Let the support set contain $\check{\mathbf{q}}_{0}$ and $\tilde{\mathbf{q}}_{0}$. The convex combination $\lambda \check{\mathbf{q}}_{0}+(1-\lambda) \tilde{\mathbf{q}}_{0}$ for $\lambda \in[0,1]$ satisfies the nonnegativity constraint and $\mathbf{p}_{0}^{\prime}\left(\lambda \check{\mathbf{q}}_{0}+(1-\lambda) \tilde{\mathbf{q}}_{0}\right)=\lambda \mathbf{x}_{0}+(1-\lambda) \mathbf{x}_{0}=\mathbf{x}_{0}$. Finally, we have $\mathbf{p}_{t}^{\prime} \check{\mathbf{q}}_{0} \geq \mathbf{p}_{t}^{\prime} \mathbf{q}_{t}\left(\tilde{x}_{t}\right)$ and $\mathbf{p}_{t}^{\prime} \tilde{\mathbf{q}}_{0} \geq \mathbf{p}_{t}^{\prime} \mathbf{q}_{t}\left(\tilde{x}_{t}\right)$ so that $\mathbf{p}_{t}^{\prime}\left(\lambda \check{\mathbf{q}}_{0}+(1-\lambda) \tilde{\mathbf{q}}_{0}\right) \geq \mathbf{p}_{t}^{\prime} \mathbf{q}_{t}\left(\tilde{x}_{t}\right)$.

Q.E.D.

Proof OF Proposition 3: If $\left\{\mathbf{p}_{t}, \mathbf{q}_{t}\right\}_{t=1,2, \ldots, T}$ fails SARP, then both sets are empty and the proposition holds trivially. In the following we shall assume that $\left\{\mathbf{p}_{t}, \mathbf{q}_{t}\right\}_{t=1,2, \ldots, T}$ passes SARP. We shall first show $S^{\mathrm{LP}} \supseteq S$, then part (ii) of the proposition, and then $\operatorname{cl}(S) \supseteq S^{\mathrm{LP}}$.

$S^{\mathrm{LP}}\left(\mathbf{p}_{0}, x_{0}\right) \supseteq S\left(\mathbf{p}_{0}, x_{0}\right)$ : Take any $\mathbf{q}_{0} \in S\left(\mathbf{p}_{0}, x_{0}\right)$. We have $\mathbf{q}_{0} \geq \mathbf{0}$ and $\mathbf{p}_{0}^{\prime} \mathbf{q}_{0}=$ $x_{0}$, and $\left\{\mathbf{p}_{t}, \mathbf{q}_{t}\right\}_{t=1,2, \ldots, T}$ satisfies SARP. Thus we only need to check the last condition in $S^{\mathrm{LP}}$. Since $\mathbf{p}_{0}^{\prime} \mathbf{q}_{0}=x_{0}=\mathbf{p}_{0}^{\prime} \mathbf{q}_{t}$, we have $\mathbf{q}_{0} R^{0} \mathbf{q}_{t}$ which implies $\mathbf{q}_{0} R \mathbf{q}_{t}$. The definition of SARP then gives $\mathbf{p}_{t}^{\prime} \mathbf{q}_{t}<\mathbf{p}_{t}^{\prime} \mathbf{q}_{0}$, which is the condition in the definition of $S^{\mathrm{LP}}\left(\mathbf{p}_{0}, x_{0}\right)$.

For part (ii) of the proposition we have

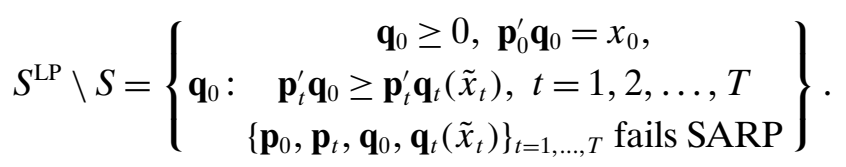


If $\mathbf{q}_{0}=\mathbf{q}_{t}\left(\tilde{x}_{t}\right), \mathbf{q}_{0} \in S$ so that we only need to consider $\mathbf{q}_{0} \neq \mathbf{q}_{t}\left(\tilde{x}_{t}\right)$ for all $t$. This and the failure of SARP imply either (A) or (B):

(A) $\mathbf{q}_{t}\left(\tilde{x}_{t}\right) R \mathbf{q}_{0}$ and $\mathbf{p}_{0}^{\prime} \mathbf{q}_{0} \geq \mathbf{p}_{t}^{\prime} \mathbf{q}_{t}\left(\tilde{x}_{t}\right)$ for some $t$. The first statement requires that there is some $s$ such that $\mathbf{q}_{s}\left(\tilde{x}_{s}\right) R^{0} \mathbf{q}_{0}$, which implies $\mathbf{p}_{s}^{\prime} \mathbf{q}_{s}\left(\tilde{x}_{s}\right) \geq \mathbf{p}_{s}^{\prime} \mathbf{q}_{0}$. Combining this with the condition $\mathbf{p}_{s}^{\prime} \mathbf{q}_{0} \geq \mathbf{p}_{s}^{\prime} \mathbf{q}_{s}\left(\tilde{x}_{s}\right)$ gives $\mathbf{p}_{s}^{\prime} \mathbf{q}_{0}=\mathbf{p}_{s}^{\prime} \mathbf{q}_{s}\left(\tilde{x}_{s}\right)$ as in the statement in the proposition.

(B) $\mathbf{q}_{0} R \mathbf{q}_{t}\left(\tilde{x}_{t}\right)$ and $\mathbf{p}_{t}^{\prime} \mathbf{q}_{t}\left(\tilde{x}_{t}\right) \geq \mathbf{p}_{0}^{\prime} \mathbf{q}_{0}$. In this case the latter statement and $\mathbf{p}_{t}^{\prime} \mathbf{q}_{0} \geq \mathbf{p}_{t}^{\prime} \mathbf{q}_{t}\left(\tilde{x}_{t}\right)$ give the statement in the proposition.

$\operatorname{cl}(S) \supseteq S^{\mathrm{LP}}$ : We have just shown that it is only the boundary of $S^{\mathrm{LP}}$ that is not in $S$. Thus the closure of $S$ contains $S^{\mathrm{LP}}$.

Q.E.D.

ProOf OF Proposition 4: Since $\mathbf{p}_{T+1}=\mathbf{p}_{0}$, we have that $S^{T+1}$ is a singleton (by part (ii) of Proposition 2). Since $S^{T}$ is convex and there are two distinct intersection points in $S^{T}$, there is a continuum of points in $S^{T}$. Hence $S^{T}$ strictly includes $S^{T+1}$.

Q.E.D.

ProOF OF PROPOSITION 5: We first show that intersection of the budget plane $\left\{\mathbf{p}_{T+1}, x_{T+1}\right\}$ with $S^{T}\left(\mathbf{p}_{0}, x_{0}\right)$ implies that $S^{T+1}\left(\mathbf{p}_{0}, x_{0}\right) \subset S^{T}\left(\mathbf{p}_{0}, x_{0}\right)$. The definition of intersection between the new budget plane $\left\{\mathbf{p}_{T+1}, x_{T+1}\right\}$ and $S^{T}\left(\mathbf{p}_{0}, x_{0}\right)$ implies that $\mathbf{q}_{T+1}\left(\tilde{x}_{T+1}\right) R^{0} \mathbf{q}_{0}$. Since $\mathbf{q}_{0} \in S^{T}\left(\mathbf{p}_{0}, x_{0}\right)$, the definition of an intersection demand implies $\mathbf{q}_{0} R^{0} \mathbf{q}_{T+1}\left(\tilde{x}_{T+1}\right)$. This gives a violation of SARP in the data set $\left\{\mathbf{p}_{t}, \mathbf{q}_{t}\left(\tilde{x}_{t}\right)\right\}_{t=0, \ldots, T+1}$. Therefore, $\mathbf{q}_{0} \notin S^{T+1}\left(\mathbf{p}_{0}, x_{0}\right)$ and hence $S^{T+1}\left(\mathbf{p}_{0}, x_{0}\right) \subset S^{T}\left(\mathbf{p}_{0}, x_{0}\right)$.

We now show that $S^{T+1}\left(\mathbf{p}_{0}, x_{0}\right) \subset S^{T}\left(\mathbf{p}_{0}, x_{0}\right)$ implies intersection of the budget plane $\left\{\mathbf{p}_{T+1}, x_{T+1}\right\}$ with $S^{T}\left(\mathbf{p}_{0}, x_{0}\right)$. Suppose $S^{T+1}\left(\mathbf{p}_{0}, x_{0}\right) \subset S^{T}\left(\mathbf{p}_{0}, x_{0}\right)$. This implies that there exists at least one $\mathbf{q}_{0} \in S^{T}\left(\mathbf{p}_{0}, x_{0}\right)$ such that $\mathbf{q}_{0} \notin S^{T+1}\left(\mathbf{p}_{0}, x_{0}\right)$. In the following discussion, $\overline{R^{0}}$ denotes "not $R^{0}$." Since $\left\{\mathbf{p}_{t}, \mathbf{q}_{t}\left(\tilde{x}_{t}\right)\right\}_{t=0, \ldots, T}$ satisfies SARP and since $\mathbf{q}_{0} R^{0}\left\{\mathbf{q}_{t}\left(\tilde{x}_{t}\right)\right\}_{t=1, \ldots, T}$ by the definition of intersection demands, this implies that $\left\{\mathbf{q}_{t}\left(\tilde{x}_{t}\right)\right\}_{t=1, \ldots, T} \overline{R^{0}} \mathbf{q}_{0}$. Since $\mathbf{q}_{0} \notin S^{T+1}\left(\mathbf{p}_{0}, x_{0}\right)$, the data set $\left\{\mathbf{p}_{t}, \mathbf{q}_{t}\left(\tilde{x}_{t}\right)\right\}_{t=0, \ldots, T+1}$ violates SARP. Given $\left\{\mathbf{q}_{t}\left(\tilde{x}_{t}\right)\right\}_{t=1, \ldots, T} \overline{R^{0}} \mathbf{q}_{0}$ and the assumption that $S^{T+1}\left(\mathbf{p}_{0}, x_{0}\right) \neq \varnothing$, this violation must result from $\mathbf{q}_{T+1}\left(\tilde{x}_{T+1}\right) R^{0} \mathbf{q}_{0} \Rightarrow$ $x_{T+1} \geq \mathbf{p}_{T+1}^{\prime} \mathbf{q}_{0}$. Hence $\mathbf{q}_{0}$ must lie in the intersection of the convex set $S^{T}\left(\mathbf{p}_{0}, x_{0}\right)$ and the closed half-space $\mathbf{p}_{T+1}^{\prime} \mathbf{q}_{0} \leq x_{T+1}$. If there exists some $\mathbf{q}_{0} \in S^{T}\left(\mathbf{p}_{0}, x_{0}\right)$ such that $\mathbf{p}_{T+1}^{\prime} \mathbf{q}_{0}<x_{T+1}$, then there must also exist some $\mathbf{q}_{0} \in S^{T}\left(\mathbf{p}_{0}, x_{0}\right)$ such that $\mathbf{p}_{T+1}^{\prime} \mathbf{q}_{0}=x_{T+1}$ and therefore the new budget plane $\left\{\mathbf{p}_{T+1}, x_{T+1}\right\}$ intersects with $S^{T}\left(\mathbf{p}_{0}, x_{0}\right)$.

Q.E.D.

\section{APPENDIX A2: DATA DESCRIPTIVES}

\section{Commodity Groups}

Food: \{bread, cereals, biscuits \& cakes, beef, lamb, pork, bacon, poultry, other meats \& fish, butter, oil \& fats, cheese, eggs, fresh milk, milk products, tea, coffee, soft drinks, sugar \& preserves, sweets \& chocolate, potatoes, other vegetables, fruit, other foods, canteen meals, other restaurant meals \& snacks\}. 
TABLE A.I

DESCRIPTIVE STATISTICS, 1975 TO 1999a

\begin{tabular}{|c|c|c|c|c|c|c|c|c|}
\hline & \multicolumn{3}{|c|}{ Budget Shares } & \multirow[b]{2}{*}{ Total Exp. } & \multicolumn{2}{|c|}{ Prices } & \multirow[b]{2}{*}{ Children } & \multirow[b]{2}{*}{$n$} \\
\hline & F & ND & $\mathrm{S}$ & & $\mathrm{F}$ & $\mathrm{S}$ & & \\
\hline 1975 & 0.3587 & 0.3166 & 0.3247 & 33.7838 & 1.0000 & 1.0000 & 1.9893 & 1873 \\
\hline 1976 & 0.3577 & 0.3076 & 0.3347 & 32.5127 & 1.0881 & 1.0687 & 1.9702 & 1642 \\
\hline 1977 & 0.3564 & 0.3124 & 0.3312 & 32.3477 & 1.1574 & 1.0447 & 1.9429 & 1770 \\
\hline 1978 & 0.3556 & 0.3136 & 0.3308 & 32.5452 & 1.1067 & 1.0398 & 1.8828 & 1681 \\
\hline 1979 & 0.3458 & 0.3196 & 0.3346 & 36.4990 & 1.1457 & 1.0414 & 1.8893 & 1689 \\
\hline 1980 & 0.3384 & 0.3208 & 0.3408 & 36.6857 & 1.1145 & 1.1061 & 1.8619 & 1781 \\
\hline 1981 & 0.3363 & 0.3061 & 0.3576 & 35.7316 & 1.1056 & 1.1836 & 1.8751 & 1906 \\
\hline 1982 & 0.3218 & 0.3101 & 0.3681 & 35.8705 & 1.1262 & 1.2199 & 1.8539 & 1876 \\
\hline 1983 & 0.3214 & 0.3129 & 0.3657 & 35.6571 & 1.0775 & 1.2429 & 1.8571 & 1743 \\
\hline 1984 & 0.3162 & 0.3151 & 0.3688 & 37.5016 & 1.1081 & 1.2492 & 1.8438 & 1671 \\
\hline 1985 & 0.3081 & 0.3207 & 0.3712 & 37.8100 & 1.0759 & 1.2242 & 1.8323 & 1622 \\
\hline 1986 & 0.3088 & 0.3221 & 0.3692 & 38.4100 & 1.0556 & 1.2239 & 1.8645 & 1587 \\
\hline 1987 & 0.3043 & 0.3228 & 0.3730 & 39.0197 & 1.0819 & 1.2372 & 1.8713 & 1632 \\
\hline 1988 & 0.3042 & 0.3278 & 0.3680 & 41.5325 & 1.0807 & 1.2512 & 1.8744 & 1648 \\
\hline 1989 & 0.3054 & 0.3222 & 0.3724 & 41.5346 & 1.0786 & 1.2713 & 1.8662 & 1652 \\
\hline 1990 & 0.3017 & 0.3129 & 0.3854 & 44.2983 & 1.1084 & 1.3150 & 1.8966 & 1538 \\
\hline 1991 & 0.2972 & 0.3103 & 0.3925 & 42.6966 & 1.0839 & 1.3207 & 1.8351 & 1510 \\
\hline 1992 & 0.2882 & 0.3121 & 0.3997 & 41.5212 & 1.0616 & 1.3445 & 1.9068 & 1578 \\
\hline 1993 & 0.2866 & 0.3077 & 0.4057 & 41.3798 & 1.0332 & 1.3533 & 1.8895 & 1511 \\
\hline 1994 & 0.2825 & 0.3029 & 0.4146 & 40.9660 & 1.0305 & 1.3748 & 1.8838 & 1489 \\
\hline 1995 & 0.2912 & 0.2912 & 0.4176 & 39.6002 & 1.0439 & 1.3645 & 1.8622 & 1502 \\
\hline 1996 & 0.2889 & 0.2999 & 0.4112 & 41.8850 & 1.0671 & 1.3491 & 1.8638 & 1476 \\
\hline 1997 & 0.2741 & 0.3041 & 0.4218 & 45.2517 & 1.0655 & 1.4071 & 1.8410 & 1421 \\
\hline 1998 & 0.2788 & 0.2981 & 0.4230 & 44.0626 & 1.0551 & 1.4102 & 1.9099 & 1432 \\
\hline 1999 & 0.2722 & 0.3032 & 0.4245 & 47.1033 & 1.0918 & 1.4367 & 1.8774 & 1501 \\
\hline
\end{tabular}

${ }^{\mathrm{a}} \mathrm{F}=$ food $\mathrm{ND}=$ nondurables; $\mathrm{S}=$ services.

Nondurables: \{beer, wine \& spirits, cigarettes, other tobacco, household consumables, pet care, men's outer clothes, women's outer clothes, children's outer clothes, other clothes, footwear, chemist's goods, audio visual goods, records \& toys, book \& newspapers, gardening goods\}.

Services: $\quad$ domestic fuels, postage \& telephone, domestic services, fees \& subscriptions, personal services, maintenance of motor vehicles, petrol \& oil, vehicle tax \& insurance, travel fares, TV licenses, entertainment $\}$.

\section{APPENDIX A3: MOMENT INEQUALITIES AND REVEALED PREFERENCE CONDITIONS}

First we show that in the current context SARP and GARP are equivalent. Since SARP implies GARP, this requires us to show that GARP implies SARP. 
For intersection demands $\tilde{\mathbf{q}}_{t}$ and $\tilde{\mathbf{q}}_{s}$ we always have that $\mathbf{p}_{t}^{\prime} \tilde{\mathbf{q}}_{t} \neq \mathbf{p}_{t}^{\prime} \tilde{\mathbf{q}}_{s}$ for any $s$ and $t$. Thus $\tilde{\mathbf{q}}_{t} \neq \tilde{\mathbf{q}}_{s}$ and $\mathbf{p}_{t}^{\prime} \tilde{\mathbf{q}}_{t}<\mathbf{p}_{t}^{\prime} \tilde{\mathbf{q}}_{s}$ if $\mathbf{p}_{t}^{\prime} \tilde{\mathbf{q}}_{t} \leq \mathbf{p}_{t}^{\prime} \tilde{\mathbf{q}}_{s}$. If we have two intersection demands $\tilde{\mathbf{q}}_{s}$ and $\tilde{\mathbf{q}}_{t}$ such that $\tilde{\mathbf{q}}_{s} R \tilde{\mathbf{q}}_{t}$, then GARP implies $\mathbf{p}_{t}^{\prime} \tilde{\mathbf{q}}_{t} \leq \mathbf{p}_{t}^{\prime} \tilde{\mathbf{q}}_{s}$. This in turn implies that the SARP conditions hold. Note that if we took demands that were not intersection demands, then we might have $\mathbf{p}_{t}^{\prime} \mathbf{q}_{t}=\mathbf{p}_{t}^{\prime} \mathbf{q}_{s}$. This is the case, for example, for the sequential maximum power (SMP) paths described in Blundell, Browning, and Crawford (2003) which specifically choose a sequence of demands with $\mathbf{p}_{t}^{\prime} \mathbf{q}_{t}=\mathbf{p}_{t}^{\prime} \mathbf{q}_{s}$. In this case the data might pass GARP but reject SARP.

We now show that the GARP conditions in Definition 3 can be recast in "standard" moment inequality restrictions (MIR) form. From Varian (1982) we have that the data $\left\{\mathbf{p}_{t}, \mathbf{q}_{t}\right\}_{t=1, \ldots, T}$ satisfy GARP if and only there exist $2 T$ scalars $V_{1}, \ldots, V_{T}$ and $\lambda_{1}, \ldots, \lambda_{T}$ such that

$$
\begin{aligned}
& V_{t}-V_{s}+\lambda_{t} \mathbf{p}_{t}^{\prime}\left(\mathbf{q}_{s}-\mathbf{q}_{t}\right) \geq 0 \text { for all } s, t, \\
& \lambda_{t} \geq 1 \text { for all } t .
\end{aligned}
$$

Given parametric intersection demands $\mathbf{q}_{t}\left(\tilde{x}_{t}\right)=\mathbf{f}\left(\boldsymbol{\theta}_{t}\right)$, define

$$
r_{t s}(\boldsymbol{\theta})=\mathbf{p}_{t}^{\prime}\left(\mathbf{f}\left(\tilde{x}_{s} ; \boldsymbol{\theta}_{s}\right)-\mathbf{f}\left(\tilde{x}_{t} ; \boldsymbol{\theta}_{t}\right)\right)
$$

Then the Afriat inequalities become

$$
\begin{aligned}
& V_{t}-V_{s}+\lambda_{t} r_{t s}(\boldsymbol{\theta}) \geq 0 \text { for all } s, t, \\
& \lambda_{t} \geq 1 \text { for all } t .
\end{aligned}
$$

In general the $V_{t}$ 's and $\lambda_{t}$ 's will not be unique for any given set of $r_{t s}$ 's. Varian (1982) provided an algorithm that takes in $T(T-1)$ values for the $r_{t s}$ 's and returns unique values for the $V_{t}$ 's and $\lambda_{t}$ 's. The mapping is continuous. Using this algorithm, the $V_{t}$ 's and $\lambda_{t}$ 's are functions of the parameters $\boldsymbol{\theta}$. The expected moment form for (15) is then

$$
\begin{aligned}
& E\left(V_{t}(\boldsymbol{\theta})-V_{s}(\boldsymbol{\theta})+\lambda_{t}(\boldsymbol{\theta}) r_{t s}(\boldsymbol{\theta})\right) \geq 0 \text { for all } s, t, \\
& E\left(\lambda_{t}(\boldsymbol{\theta})\right)-1 \geq 0 \quad \text { for all } t,
\end{aligned}
$$

which is of the MIR form given in Andrews and Guggenberger (2007).

\section{APPENDIX A4: EMPIRICAL RESULTS USING A PARAMETRIC QUADRATIC ENGEL CURVE SPECIFICATION FOR EXPANSION PATHS}

In this appendix we present a comparison of the semiparametric Engel curve analysis used for the empirical results presented in Section 5 with a parametric analysis based on the quadratic Engle curve specification underlying the QUAIDS model (see Banks, Blundell, and Lewbel (1997)). Figure 14 com- 


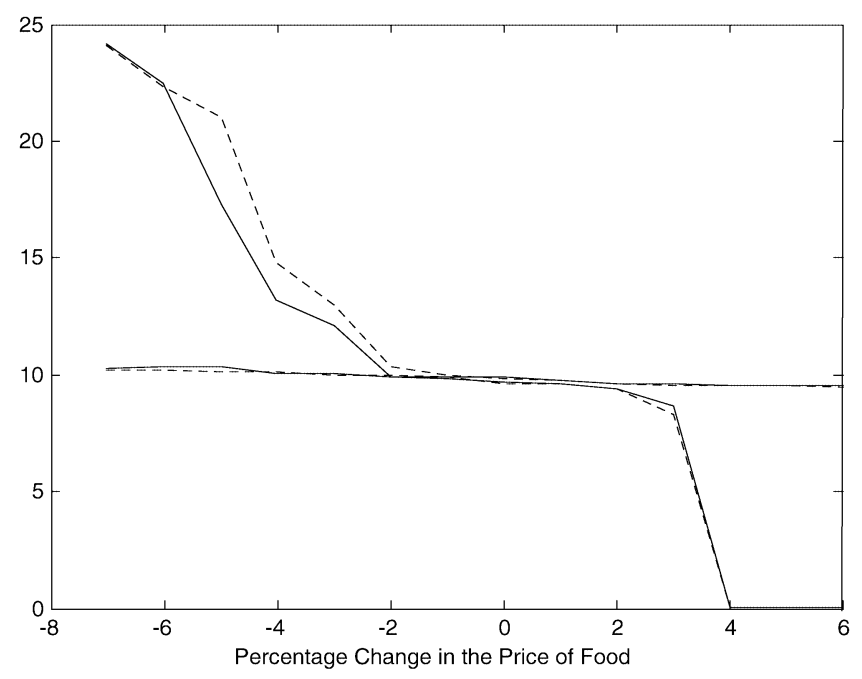

FIGURE 14.-Constrained E-bounds for food: Nonparametric and parametric Engel curves.

pares the estimated E-bounds on the demand curve. The dashed bounds are for the semiparametric estimates and are the same as the dashed bounds in Figure 8; they used the data from all of the periods together, are based on the semiparametric Engel curve estimates described in Section 3.2.1, and impose the RP conditions on the intersection demands as discussed in Section 5.1. The solid bounds are those derived from the same procedure applied to parametric (quadratic) Engel curves estimated from the same data and with the RP conditions similarly imposed.

We note that the bounds derived from the parametric Engel curve model are very similar to those from the nonparametric model, and show the same characteristic widening and tightening according to the local density of the price data. We also conduct a statistical test of the RP conditions for the parametrically estimated intersection demands (identical to the one we describe in the text for the semiparametric estimates) and, like the test based on the semiparametric estimates, the data reject RP with a $p$-value very close to zero. This is not surprising given the relative precision of parametric versus nonparametric estimates.

As in the semiparametric case in the text, we conducted a search for a set of years which did not reject RP in the parametric model and derived the bounds from those intersection demands. The result is shown in Figure 15, which is the bound recovered from the years $\{78,79,84-87,93,94\}$, which give a $p$-value on the RP test of 0.03 . The solid bounds are those for all of the years (the same as Figure 14), whilst the dashed bounds are from the RP-consistent subset.

As in the semiparametric case, using fewer years of data widens the bounds, although the years which remain are informative enough that the bounds 


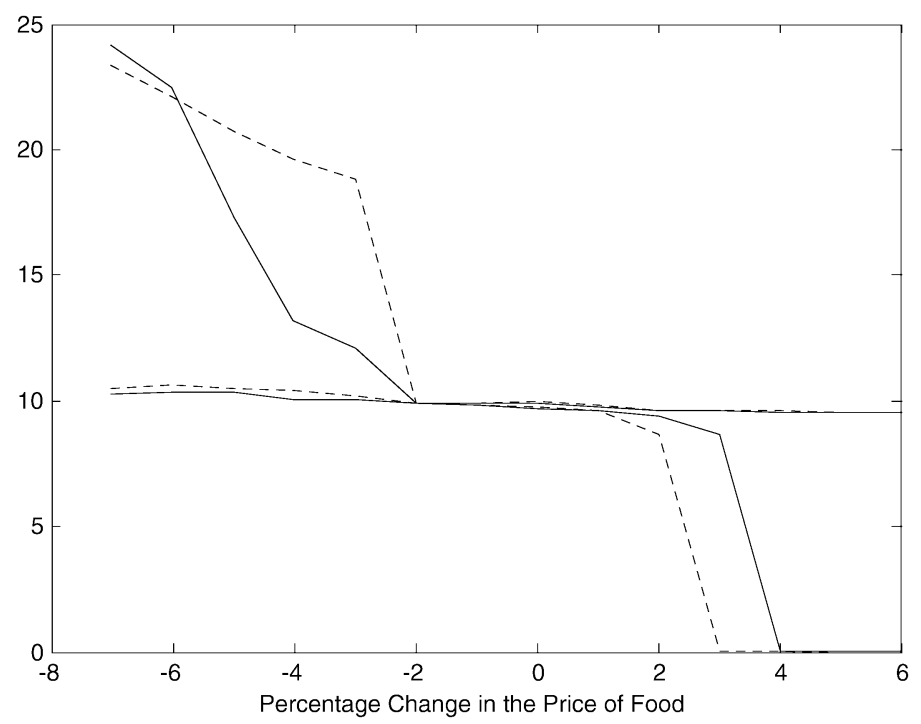

FIGURE 15.-Constrained E-bounds for food: Parametric Engel curves for all periods and an RP-consistent subset.

remain tight over a good range of relative price changes. Figure 16 illustrates the bounds for the RP-consistent subset of period alone.

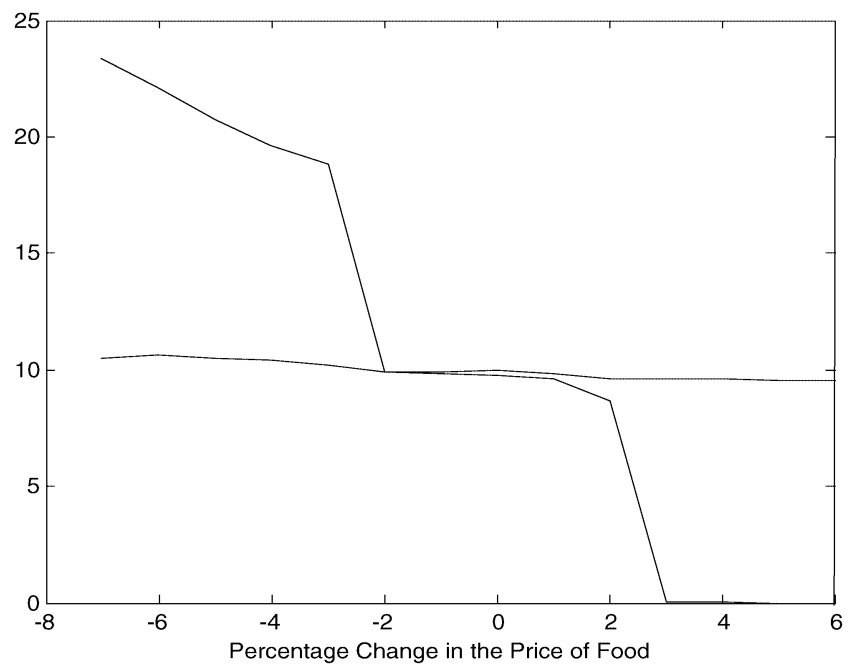

FIGURE 16.-Constrained E-bounds for food: Parametric Engel curves using an RP-consistent subset. 


\section{REFERENCES}

Afriat, S. N. (1973): "On a System of Inequalities in Demand Analysis: An Extension of the Classical Method," International Economic Review, 14, 460-472. [1228]

ANDREWS, D. W. K. (1995): "Nonparametric Kernel Estimation for Semiparametric Models," Econometric Theory, 11, 560-596. [1241,1242]

ANDREws, D. W. K., AND P. GugGenBerger (2007): "Validity of Subsampling and 'Plug-In' Asymptotic Inference for Parameters Defined by Moment Inequalities," Discussion Paper 1620, Cowles Foundation. [1246,1247,1258]

BANKS, J., R. W. BLundell, AND A. LEWBel (1996): "Tax Reform and Welfare Measurement: Do We Need Demand System Estimation?” Economic Journal, 106, 1227-1241. [1230]

(1997): "Quadratic Engel Curves, Indirect Tax Reform and Welfare Measurement," Review of Economics and Statistics, 79, 527-539. [1258]

BECKERT, W., AND R. W. BLUNDELL (2005): "Invertibility of Nonparametric Stochastic Demand Functions," Working Paper CWP09/05, Cemmap, forthcoming in Review of Economic Studies. [1243]

BLUNDELl, R., AND A. DUNCAN (1998): "Kernel Regression in Empirical Microeconomics," Journal of Human Resources, 33, 62-87. [1240]

BLUNDELL, R., AND M. J. POWELL (2003): "Endogeneity in Nonparametric and Semiparametric Regression Models," in Advances in Economics and Econometrics, ed. by M. Dewatripont, L. Hansen, and S. J. Turnovsky. Cambridge, U.K.: Cambridge University Press, ESM 36, Chap. 3, 312-357. [1241,1242]

Blundell, R., M. BROWNing, AND I. CRAWFORD (2003): "Nonparametric Engel Curves and Revealed Preference," Econometrica, 71, 205-240. [1227-1229,1240,1258]

(2007): "Improving Revealed Preference Bounds on Demand Responses," International Economic Review, 48, 1227-1244. [1237]

(2008): "Supplements to 'Best Nonparametric Bounds on Demand Responses'," Econometrica Supplementary Material, 76, http://www.econometricsociety.org/ecta/Supmat/6096_ data-1.zip; http://www.econometricsociety.org/ecta/Supmat/6096_data-2.zip; http://www. econometricsociety.org/ecta/Supmat/6096_data-3.zip; http://www.econometricsociety.org/ecta/ Supmat/6096_data and programs.zip; http://www.econometricsociety.org/ecta/Supmat/6096_ data description.pdf. [1231]

Blundell, R., X. CHEN, AND D. KRISTENSEN (2007): "Semi-Nonparametric IV Estimation of Shape-Invariant Engel Curves," Econometrica, 75, 1613-1669. [1241]

BLundell, R., A. DunCAN, AND K. PendaKuR (1998): "Semiparametric Estimation and Consumer Demand," Journal of Applied Econometrics, 13, 435-461. [1240,1242]

Blundell, R., P. Pashardes, AND G. Weber (1993): "What Do We Learn About Consumer Demand Patterns From Micro Data?” American Economic Review, 83, 570-597. [1230]

BROWn, D. J., AND R. L. MATZKIN (1998): "Estimation of Simultaneous Equations Models, With an Application to Consumer Demand," Discussion Paper, Cowles Foundation. [1243]

BROWNING, M., AND P.-A. CHIAPPORI (1998): "Efficient Intra-Household Allocations: A General Characterization and Empirical Tests," Econometrica, 66, 1241-1278. [1230]

BurniauX, J.-M., T.-T. Dang, D. Fore, M. F. Förster, M. Mira D’Ercole, and H. OxLey (1998): "Income Distribution and Poverty in Selected OECD Countries," Working Paper 189, Economics Department, OECD, Paris. [1240]

Cherchye, L., B. De Rock, AND F. Vermeulen (2007): "The Collective Model of Household Consumption: A Nonparametric Characterization,” Econometrica, 75, 553-574. [1230]

ChernozhukOV, V., H. HONG, AND E. TAMER (2007): "Estimation and Confidence Regions for Parameter Sets in Econometric Models," Econometrica, 75, 1243-1284. [1246,1247]

Deaton, A. S., AND J. Muellbauer (1980): "An Almost Ideal Demand System," American Economic Review, 70, 312-336. [1230,1243]

HAUSMAN, J., W. NEWEY, H. ICHIMURA, AND J. POWELl (1991): "Identification and Estimation of Polynomial Errors-in-Variables Models," Journal of Econometrics, 50, 273-295. [1230] 
IMBENS, G., AND W. NEWEY (2007): "Identification and Estimation of Triangular Simultaneous Equations Models Without Additivity," Mimeo, MIT. [1243]

JoRGENSON, D. W., L. J. LAU, AND T. M. STOKER (1982): "The Transcendental Logarithmic Model of Aggregate Consumer Behavior," in Advances in Econometrics, Vol. 1, ed. by R. Basmann and G. Rhodes. Greenwich, CT: JAI Press. [1230]

LEwBel, A. (1991): "The Rank of Demand Systems: Theory and Nonparametric Estimation," Econometrica, 59, 711-730. [1230]

(2001): "Demand Systems With and Without Errors: Reconciling Econometric, Random Utility and GARP Models," American Economic Review, 91, 611-618. [1243]

MANSKI, C. F. (2003): Partial Identification of Probability Distributions, Springer Series in Statistics, Vol. 12. Berlin: Springer Verlag. [1229,1246]

(2007): Identification for Prediction and Decision. Cambridge, MA: Harvard University Press. [1228]

MATZKIN, R. L. (2007): "Heterogeneous Choice," in Advance in Econometrics: Proceedings of the 9th World Congress, Vol. III, Econometric Society Monograph Series, ed. by R. Blundell, W. Newey, and T. Persson. Cambridge, U.K.: Cambridge University Press. [1240,1243]

Newey, W., Powell, J., AND F. Vella (1999): "Nonparametric Estimation of Triangular Simultaneous Equations Models," Econometrica, 67, 565-604. [1241,1242]

Robinson, P. (1988): "Root- $N$-Consistent Semiparametric Regression," Econometrica, 56, 931-954. [1241,1242]

VARIAN, H. (1982): "The Nonparametric Approach to Demand Analysis," Econometrica, 50, 945-974. [1228,1232,1233,1258]

- (1983): "Nonparametric Tests of Consumer Behaviour," Review of Economic Studies, 50, 99-110. [1229]

(1986): "Nonparametric Analysis of Optimizing Behavior With Measurement Error," Journal of Econometrics, 30, 445-459. [1246]

Dept. of Economics, University College London, Gower Street, London WC1 E6BT, U.K. and Institute for Fiscal Studies, London, U.K.; r.blundell@ucl.ac.uk, Dept. of Economics, Oxford University, Oxford, OX1 3UQ and Institute for Fiscal Studies, London, U.K.; Martin.Browning@economics.ox.ac.uk,

$$
\text { and }
$$

Dept. of Economics, Oxford University, Oxford, OX1 3UQ and Institute for Fiscal Studies, London, U.K.; ian.crawford@economics.ox.ac.uk.

Manuscript received October, 2005; final revision received December, 2007. 